\title{
AUFSÄTZE
}

\section{Die Bundestagswahl vom 22. September 2013: Merkels Meisterstück}

\author{
Richard Hilmer und Stefan Merz
}

\section{Das Wahlergebnis}

Die Bundestagswahl 2013 hatte einen klaren Gewinner, brachte aber keine eindeutige Mehrheit hervor. Zum Sieger kürten die Wähler die Union, die um 7,7 Prozentpunkte zulegte und mit 41,5 Prozent erstmals seit 1994 wieder die 40-Prozent-Marke übertraf. Die Rückkehr zu alter Stärke war insbesondere für Angela Merkel eine Genugtuung, denn bei den beiden Wahlen zuvor musste sie als Spitzenkandidatin herbe Stimmenverluste verantworten. Zwar steuerte die CSU mit dem höchsten Landesergebnis von 49,3 Prozent in Bayern zum Erfolg der Union bei; da der Zuwachs bei der CDU aber größer ausfiel, stieg ihr Gewicht innerhalb der Union. Der FDP, dem alten und auch für die kommende Wahlperiode auserkorenen Wunschpartner der Union, entzogen die Wähler dagegen das Vertrauen und ließen die Liberalen an der Fünf-Prozent-Marke scheitern. Dem bislang bei weitem höchsten Wahlergebnis der FDP 2009 folgte so 2013 der Absturz. Der erzielte Stimmenanteil von 4,8 Prozent bedeutet das historisch schlechteste Ergebnis der FDP.

Deutliche Einbußen gegenüber 2009 mussten auch die Linke $(-3,3)$ und die Grünen $(-2,3)$ hinnehmen, ihr Wiedereinzug war jedoch mit Stimmenanteilen von 8,6 beziehungsweise 8,4 Prozent zu keinem Zeitpunkt gefährdet. Bei der Linken kamen die Verluste nicht überraschend, letztlich fielen sie sogar geringer aus als lange Zeit befürchtet. Da sie trotz der Verluste sogar erstmals stärkste Oppositionspartei wurde, fühlte sich das Ergebnis wie ein Sieg an. Bei den Grünen verhielt es sich genau umgekehrt: Sie rechneten fest mit Stimmengewinnen, fielen aber hinter ihr historisch bestes Ergebnis von 2009 und damit wieder in das gewohnte Auf und Ab bei Bundestagswahlen zurück.

Bei der SPD erfüllten sich zwar die Erwartungen an eine Trendumkehr nach drei aufeinander folgenden Wahlen mit Stimmenverlusten, die 2009 in einem Debakel und dem bislang schlechtesten Wahlergebnis von 23 Prozent geendet hatten. Der Zuwachs von 2,7 Prozentpunkten blieb jedoch weit hinter ihren Erwartungen zurück. Der Rückstand der SPD zur Union wuchs sogar auf 15,8 Punkte an - der größte Abstand zwischen den beiden Volksparteien seit 1957.

Zu den Wahlgewinnern darf sich auch die AfD rechnen, die bei ihrer ersten Bundestagswahl mit 4,7 Prozent die Mandatsschwelle nur knapp verfehlte. Gegenüber 2009 leicht zulegen konnten zwar auch die Piraten $(+0,2)$, deren Ergebnis von 2,2 Prozent blieb aber nach den Erfolgen bei den Landtagswahlen 2011 und 2012 weit unter ihren Erwartungen. Die NPD verlor auch nach dem förmlichen Zusammenschluss mit der DVU zum dritten Mal in Folge an Stimmen (-0,2) und kam auf 1,3 Prozent. Die erstmals bei einer Bundestagswahl angetretenen Freien Wähler erzielten einen Stimmenanteil von einem Prozent. Die übrigen 20 angetretenen Parteien blieben ausnahmslos deutlich darunter. 
Mit dem ersten Anstieg der Wahlbeteiligung seit 1998 wurde der seit der Bundestagswahl 1976 anhaltende, nur zwei Mal unterbrochene Trend einer von Wahl zu Wahl sinkenden Beteiligung gestoppt. Allerdings wäre es voreilig, bei einem Anstieg von gerade einmal 0,7 Punkten von einer Trendumkehr zu sprechen. Mit 71,5 Prozent war die zweitschlechteste Beteiligung bei einer Bundestagswahl zu verzeichnen, was immer noch gut sechs Punkte unter der von 2005 liegt, der drittschlechtesten in der deutschen Wahlgeschichte.

Insgesamt schafften diesmal nur vier Parteien (CDU und CSU als eine Partei betrachtet) den Einzug in den neuen Bundestag, in dem die Union nunmehr drei Parteien aus dem linken Spektrum gegenübersteht. Wegen unüberwindlicher Gegensätze, etwa in der Außenund Steuerpolitik, erwies sich diese linke Mehrheit aber als nicht koalitionsfähig. Als klarer Sieger hatte die Union am Ende die Wahl zwischen einem Bündnis mit der SPD oder mit den Grünen. Beinahe hätte sie keinen Partner gebraucht, denn sie verfehlte die absolute Mehrheit nur denkbar knapp, da diesmal wegen der vielen bei der Sitzverteilung unberücksichtigten Stimmen etwas über 42 Prozent für eine absolute Mehrheit der Mandate ausgereicht hätte. Mit 41,5 Prozent kamen CDU und CSU dieser Schwelle sehr nahe - am Ende fehlten gerade einmal fünf Sitze.

Mit diesem Wahlergebnis wurde die steigende Bereitschaft der Wähler zum Parteiwechsel bestätigt. Jeder dritte Wähler von 2009 gab diesmal einer anderen Partei die Zweitstimme als vier Jahre zuvor ${ }^{1}, 2009$ lag der Anteil der Parteiwechsler noch bei 31 Prozent. $^{2}$ Die Wählerströme nahmen diesmal allerdings eine völlig andere Richtung, denn die Gewinner von 2009 verloren bei der Bundestagswahl 2013 ausnahmslos an Stimmen, wobei das Scheitern der FDP eine Zäsur in der deutschen Wahlgeschichte darstellt, gehörten die Liberalen doch zum festen Inventar des deutschen Parteienwesens seit 1949. Es war die FDP, die im alten Westdeutschland die Regierungswechsel herbeiführte, weil sie als mit Abstand kleinste Partei in den Parlamenten seit 1949 als Zünglein an der Waage fungierte und abwechselnd Union und SPD zu Mehrheiten verhalf. Es dauerte bis 1998, bis der erste Regierungswechsel ohne Mithilfe der FDP zustande kam. Ohne Präsenz im Bundestag und der Funktion des unverzichtbaren Mehrheitsbeschaffers verlustig gegangen, wird eine Rückkehr der Liberalen in den Bundestag nicht einfach sein. Von den Verlusten der kleinen Bundestagsparteien haben zum einen die beiden großen profitiert, die beide - wenn auch in sehr unterschiedlichem Ausmaß - zulegten, was letztmals 1965 der Fall war. Zumindest vorerst gestoppt wurde damit der seit den 1970er Jahren unaufhaltsam scheinende Trend des Bedeutungsschwunds der Volksparteien (vgl. Abbildung 1). Der gemeinsame Anteil von Union und SPD stieg deutlich von 56,8 auf 67,3 Prozent, blieb damit aber noch unter dem Wert von $2005(69,4)$.

Deutlich zugelegt haben aber auch die Kleinstparteien, deren Anteil sich fast verdoppelte. Anders als 2009 machte die Wechselbereitschaft nicht an den Grenzen des seit der Vereinigung etablierten Fünfparteiensystems halt - eine vergleichbare Fragmentierung des Wählervotums gab es zuletzt 1957. In der deutschen Wahlgeschichte einzigartig ist dabei das Abschneiden der AfD, denn noch nie gelang einer neu gegründeten Partei ein vergleichbarer Erfolg. Das knappe Scheitern von AfD und FDP an der Mandatsschwelle von

1 Vgl. Infratest dimap, Wahlreport Bundestagswahl. Eine Analyse der Wahl vom 22. September 2013, Berlin 2013, S. 14.

2 Vgl. Richard Hilmer, Bundestagswahl 2009: Ein Wechsel auf Raten, in: ZParl, 41. Jg. (2010), H. 1, S. 147 - 180, S. 149. 


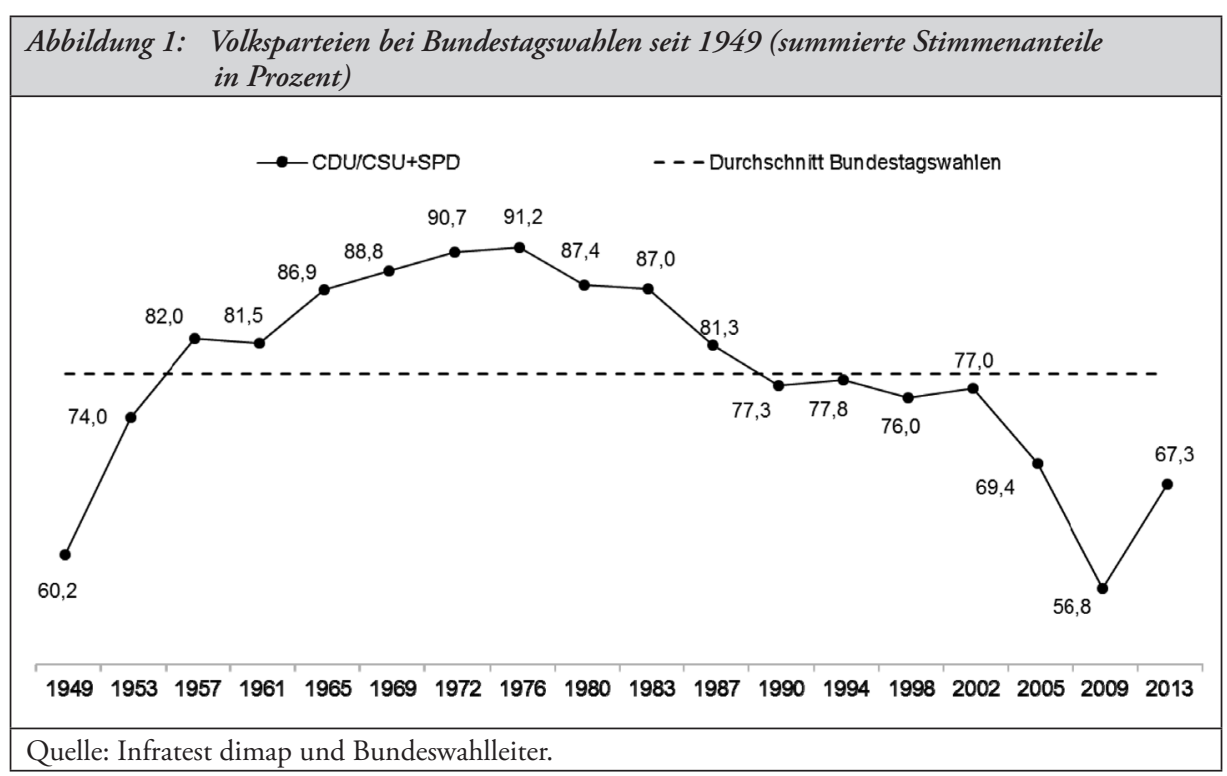

fünf Prozent lässt allerdings erahnen, wie fragil das neue Vierparteiensystem ist. Viel hätte nicht gefehlt, und es wären erstmals sechs Parteien im Bundestag vertreten gewesen. So blieb diesmal fast ein Siebtel der abgegebenen Zweitstimmen bei der Sitzverteilung unberücksichtigt. Die einen sehen darin einen Anlass, die Fünf-Prozent-Hürde in Frage zu stellen. Andere halten sie für wertvoller denn je, weil ein Absenken der Mandatsschwelle die Volatilität wohl eher noch befördern als bremsen würde und damit stabile Regierungsmehrheiten noch schwieriger zu erreichen wären.

\section{Rückblick auf die 17. Wahlperiode}

Als sich am 27. September 2009 bereits in den 18-Uhr-Prognosen eine deutliche Mehrheit für Schwarz-Gelb abzeichnete, war spätestens klar, dass nun zusammenkam, was zumindest nach Überzeugung der drei Protagonisten CDU, CSU und FDP bereits 2005 zusammengehört hätte. ${ }^{3}$ Der jeweilige Beitrag der drei Partner zum Wahlsieg fiel sehr unterschiedlich aus, denn den deutlichen Zugewinnen der FDP standen leichte Verluste von CDU und CSU gegenüber. Insgesamt stellte die FDP fast ein Drittel der Sitze in der Koalition und war damit mehr als doppelt so stark wie die CSU - in den schwarz-gelben Regierungen früherer Jahre begegneten sich Liberale und Christsoziale in der Regel auf Augenhöhe. ${ }^{4}$ Diese veränderte Binnenstatik sollte sich in der Folgezeit als schwere Hypothek für den Zusammenhalt in der Koalition erweisen.

3 Vgl. ebenda.

4 Einzige Ausnahme war die Koalition 1990, da die FDP aus der ersten gesamtdeutschen Wahl, angeführt von dem im Osten populären Hans-Dietrich Genscher, mit einem Stimmenanteil von elf Prozent die auf Bayern beschränkte CSU deutlich überflügelte. 
Die Koalitionsverhandlungen standen aufgrund anstehender internationaler Termine unter Zeitdruck und verliefen rasch und eher geräuschlos. Sie mündeten in einen 124 Seiten umfassenden Koalitionsvertrag, der nach nur drei Wochen unterzeichnet wurde. Er trug den Titel „Wachstum, Bildung, Zusammenhalt“ und enthielt einige konkrete Beschlüsse sowie eine ganze Reihe von Absichtserklärungen und Prüfaufträgen. Anstatt erhoffter Harmonie taten sich schnell grundlegende Differenzen auf, die sich auch in eilig einberufenen Klärungsrunden nicht auflösen ließen und in „dilatorischen Formelkom-promissen “5 endeten. Die Liberalen stießen vor allem mit ihrer Forderung nach Steuererleichterung auf Vorbehalte in der Union, die - wie die Mehrheit der Bevölkerung auch - der Haushaltskonsolidierung mit dem Hinweis auf die Rekordverschuldung der öffentlichen Haushalte nach der Bankenkrise den Vorrang gab. Am Ende blieben von dem FDP-Versprechen „Mehr Netto vom Brutto“ nur die Senkung der „Hotelsteuer“ übrig, die aufgrund einer hohen Spende der Mövenpick-Kette an die FDP einen schalen Beigeschmack bekam. Sehr früh war von einem „schwarz-gelben Fehlstart"6 die Rede, unter dem vor allem das Erscheinungsbild der FDP litt.

Auch die Ressortverteilung sorgte für einige Überraschungen. Angesichts des zentralen Stellenwerts der Steuerpolitik im FDP-Wahlprogramm war vielfach erwartet worden, dass die FDP das Finanzministerium für sich beanspruchen würde. Da aber Guido Westerwelle das Außenministerium bevorzugte, musste sich die FDP mit dem mit weit weniger Machtfülle ausgestatteten Wirtschaftsministerium bescheiden. Auch ansonsten folgte die Verteilung der klassischen Ressorts dem Muster früherer konservativ-liberaler Regierungen. Das Außenamt und das Justizministerium gingen wieder an die FDP, als Zugabe bekamen die erstarkten Liberalen außerdem das Gesundheits- und das Entwicklungsministerium, für dessen Abschaffung die FDP sich noch im Wahlkampf eingesetzt hatte. Die CDU stellte neben der Kanzlerin weitere sieben Minister, unter anderem in den wichtigen Ressorts Finanzen und Inneres. Der kleinste Partner, die CSU, bekam drei Ministerien zugesprochen, davon mit dem Verteidigungsministerium nur ein klassisches, das allerdings mit KarlTheodor zu Guttenberg sehr prominent besetzt wurde.

Die Wahlperiode begann im ersten Halbjahr mit einer gewissen politischen Selbstblockade, um durch bundespolitische Maßnahmen die Wahlchancen der schwarz-gelben Landesregierung in Nordrhein-Westfalen im Frühjahr 2010 nicht zu schmälern, was missglückte. Die Wahl ging verloren und damit auch die schwarz-gelbe Mehrheit im Bundesrat. Darüber hinaus war die erste Hälfte der Amtszeit der neuen Regierung gekennzeichnet durch anhaltende Querelen. Die Binnenatmosphäre wurde bereits unmittelbar nach den Koalitionsverhandlungen auf eine erste Probe gestellt, als Westerwelle vor der Presse verkündete: „Wir haben uns in allem durchgesetzt. “7 Vor allem FDP und CSU zankten sich über den steuer- und gesundheitspolitischen Kurs, wobei die Kontrahenten auch mit Schimpfwörtern nicht geizten („Gurkentruppe“, „Wildsau“8). Das Verhältnis wurde weiter belastet,

5 Thomas Saalfeld, Regierungsbildung 2009: Merkel II und ein unvollständiger Koalitionsvertrag, in: ZParl, 41. Jg. (2010), H. 1, S. 181 - 206, S. 200.

6 Jan Dams, Schwarz-gelber Fehlstart, in: Die Welt vom 3. Dezember 2009, S. 3.

7 Günter Bannas / Peter Carstens, Wir haben uns in allem durchgesetzt, FDP billigt Koalitionsvertrag, in: FAZ vom 26. November 2009, S. 1.

8 Joachim Schucht, CSU und FDP - Das Niveau der Beleidigungen sinkt, in: Die Welt vom 8. Juni 2010, http://www.welt.de/politik/deutschland/article7957025/CSU-und-FDP-Das-Niveau-derBeleidigungen-sinkt.html (Abruf am 13. Januar 2014). 
weil der CSU-Verteidigungsexperte Guttenberg auch außenpolitisch eine gute Figur machte, während Westerwelle Anlaufschwierigkeiten hatte, die sehr unterschiedlichen Anforderungen als Außenminister und als Chef einer zunehmend unter Druck geratenden FDP in Einklang zu bringen. Dieser Spagat fand seinen Höhepunkt in seinem Spruch von der „spätrömischen Dekadenz“ als Replik auf ein Urteil des Bundesverfassungsgerichts zur Neufestsetzung der Hartz-IV-Sätze. ${ }^{?}$

Überschattet wurde die erste Hälfte der Wahlperiode auch durch eine Reihe prominenter Rücktritte. Eine erste Umbesetzung im Kabinett war schon nach sechs Wochen nötig, als dem vom Verteidigungs- in das Arbeitsministerium gewechselten Franz Josef Jung die Kunduz-Affäre ${ }^{10}$ zum Verhängnis wurde - kürzer war zuvor noch kein Bundesminister im Amt gewesen. Sein Ministerium übernahm Ursula von der Leyen, der im Amt der Familienministerin die aus Hessen stammende junge Abgeordnete Kristina Köhler folgte. 2011 musste der zum neuen Star in der CSU avancierte zu Guttenberg wegen des Vorwurfs des Plagiats bei der Abfassung seiner Doktorarbeit ${ }^{11}$ zurücktreten, was ein Revirement innerhalb der Union zur Folge hatte: Das Verteidigungsministerium wechselte zur CDU und wurde dem Ex-Innenminister Lothar de Maiziére übertragen. Sein Amt übernahm der CSU-Landesgruppenchef Hans-Peter Friedrich. Auch bei den Liberalen wurde wenig später ein Ringtausch nötig, ausgelöst durch den Rücktritt Westerwelles als Parteivorsitzender und stellvertretender Regierungschef. In beiden Ämtern wurde er von Philipp Rösler beerbt, der zudem von Rainer Brüderle das Wirtschaftsministerium übernahm. Brüderle löste die Fraktionsvorsitzende Birgit Homburger ab, und Ex-Staatssekretär Daniel Bahr wurde an die Spitze des Gesundheitsressorts berufen. In der zweiten Hälfte der Wahlperiode folgten zwei weitere Rücktritte von CDU-Ministern. Auf Drängen der Kanzlerin musste Norbert Röttgen nach einer deftigen Wahlniederlage in Nordrhein-Westfalen sein Amt als Umweltminister aufgeben; ihm folgte Peter Altmaier. Und 2013 sah sich Bildungsministerin Annette Schavan zum Rücktritt gezwungen, ebenfalls wegen eines Plagiatsvorwurfs; ihren Posten übernahm Johanna Wanka.

Geprägt wurde die zweite Amtszeit Merkels auch von zwei ungeplanten Wechseln im Amt des Bundespräsidenten. Der 2004 mit den Stimmen von Union und FDP gewählte und 2009 wiedergewählte Horst Köhler legte im Mai 2010 völlig überraschend sein Amt nieder - ein weiteres Novum in der Geschichte der Bundesrepublik. Als Grund für seinen Rücktritt gab er die Kritik aus Regierungs- und Oppositionskreisen an seinen Äußerungen zu Auslandseinsätzen der Bundeswehr an. Union und FDP einigten sich rasch auf den niedersächsischen Ministerpräsidenten Christian Wulffals gemeinsamen Kandidaten, der sich allerdings erst im dritten Wahlgang gegen den Kandidaten von SPD und Grünen, Joachim Gauck, durchsetzte. Im Dezember 2011 musste sich Wulff einer Reihe von Vorwürfen erwehren, die vor allem seine frühere Amtsführung als Ministerpräsident betrafen. Nach nicht einmal

9 Vgl. Spiegel Online, Hartz-IV-Debatte: Westerwelle warnt vor Vollversorgerstaat, 11. Februar 2010, http://www.spiegel.de/politik/deutschland/hartz-iv-debatte-westerwelle-warnt-vor-vollversorgerstaat-a-677163.html (Abruf am 13. Januar 2014).

10 Bei einer von einem Bundeswehroffizier am 4. September 2009 veranlassten Beschießung von zwei von den Taliban entführten Tanklastwagen kamen auch viele Zivilisten, darunter auch Kinder, ums Leben.

11 Karl-Theodor zu Guttenberg wurde vorgeworfen, weite Teile seiner Dissertation ohne Kennzeichnung abgeschrieben zu haben, wobei ihm die einberufene Prüfungskommission seiner Universität Täuschungsvorsatz unterstellte. 
zweijähriger Amtszeit sah er sich schließlich im Februar 2012 wegen des Verdachts der Vorteilsnahme zum Rücktritt gezwungen. Bei der Suche nach einem neuen Kandidaten sprach sich Merkel für einen Bewerber aus, der auch die Zustimmung von SPD und Grünen haben sollte. Der letztlich erfolgreiche Kandidat, Joachim Gauck, wurde allerdings gegen den Willen Merkels auf Initiative einer ungewöhnlichen Koalition aus SPD, Grünen und der FDP vorgeschlagen, am Ende aber auch mit den Stimmen der Union im März 2012 gewählt. Der überraschende Alleingang der FDP bei der Kandidatensuche ohne Absprache mit den Koalitionspartnern CDU und CSU trug nicht zur Festigung der ohnehin als zerstritten wahrgenommenen Koalition bei, zumal ein etwas unglücklich geratenes erklärendes Interview des jungen FDP-Chefs dessen Verhältnis zur Bundeskanzlerin zusätzlich belastete. ${ }^{12}$

Der holprige Start der Koalition drückte sich in drastisch sinkenden Zustimmungswerten für die Regierungsarbeit aus. Im Juni 2010 zeigten sich noch ganze 12 Prozent der Bürger zufrieden mit der Bundesregierung, 86 Prozent waren unzufrieden ${ }^{13}$ - eine vergleichbare Ablehnung hatte bislang nur die rot-grüne Koalition unmittelbar nach der Verabschiedung von Hartz IV erfahren. Bis zum Frühsommer stürzte die FDP in der Wählergunst regelrecht ab und erreichte im Juni 2010 die Fünf-Prozent-Marke; die Unionsparteien verloren ebenfalls leicht und lagen im Sommer bei 31 Prozent. Selbst die Kanzlerin blieb nicht verschont von dem Stimmungstief: Ihre Zustimmungswerte sanken zwischen Dezember 2009 und Juli 2010 von 70 auf 41 Prozent. Im Herbst zog sie die Konsequenz und rief in der Generaldebatte des Bundestages den „Herbst der Entscheidungen“ aus. Von den drei Eckpunkten dieses Programms - Anpassung der Hartz-IV-Sätze, Festschreibung der Arbeitgeberbeiträge zur Krankenversicherung und Verlängerung der Laufzeiten von Atomkraftwerken - fand nur ersterer mehrheitliche Zustimmung in der Bevölkerung. ${ }^{14}$ Die Entlastung der Arbeitgeber bei der Beitragszahlung förderte das Gefühl wachsender sozialer Ungerechtigkeit, und der Schwenk in der Atompolitik gab der Anti-Atomkraftbewegung neuen Auftrieb. Viel Zuspruch in der Bevölkerung gab es dagegen für die von zu Guttenberg propagierte und letztlich auch durchgesetzte Aussetzung der Wehrpflicht. Innerhalb der Union war dieser - vor der Wahl noch kategorisch ausgeschlossene - Schritt höchst umstritten. Der Verteidigungsminister verstand es allerdings weit besser als sein Vorgänger, den Wählern den Afghanistaneinsatz der Bundeswehr - auch medial - zu vermitteln und avancierte bis Ende 2010 zum mit Abstand beliebtesten Politiker in Deutschland. Seine Zustimmungswerte fielen zeitweise fast doppelt so hoch aus wie die der Kanzlerin, was seinen Sturz am 3. März 2011 aber nicht verhindern konnte. Die Folgen seines Rücktritts hielten sich für die Regierung nicht zuletzt deshalb in Grenzen, weil eine Woche später - in der heißen Phase der Landtagswahlkämpfe in Baden-Württemberg und Rheinland-Pfalz die Nuklearkatastrophe im japanischen Fukushima die Schlagzeilen beherrschte.

Merkels Reaktion auf dieses Ereignis war für Parteifreunde, politische Gegner und auch für die Wirtschaft völlig überraschend. Sie setzte den Beschluss zur Verlängerung der Lauf-

12 Vgl. Majid Sattar, Merkel lässt Röslers Frosch-Vergleich zurückweisen, in: FAZ vom 24. Februar 2013, http:/www.faz.net/aktuell/politik/inland/nach-gauck-nominierung-merkel-laesst-roeslersfrosch-vergleich-zurueckweisen-11661182.html (Abruf am 13. Januar 2014).

13 Vgl. Infratest dimap, ARD-DeutschlandTREND Extra, KW24/2010, Berlin 2010. Im Dezember 2009 waren immerhin noch 33 Prozent mit der neu gebildeten Bundesregierung zufrieden. Vgl. Infratest dimap, ARD-DeutschlandTREND Dezember 2009, Berlin 2009, S. 10.

14 Vgl. Infratest dimap, ARD-DeutschlandTREND Oktober 2010, Berlin 2010, S. 7. 
zeiten von Atomkraftwerken aus und rief in einer Regierungserklärung am 9. Juni 2011 die „Energiewende“ aus. Das Debakel für die CDU bei der Landtagswahl in Baden-Württemberg konnte diese Ausstiegsentscheidung ebenso wenig verhindern wie die Ablösung des als vehementen Befürworter der Atomenergie geltenden CDU-Ministerpräsidenten Stefan Mappus durch den ersten grünen Chef einer Landesregierung, Winfried Kretschmann. Der Schritt verstärkte zudem den Eindruck, die Union sei „normativ entkernt“ ${ }^{15}$, und verhalf weder Merkel noch der Union kurzfristig zu höheren Zustimmungswerten. Gleichwohl erwies er sich langfristig als erfolgreich, denn er entschärfte ein auch in der Anhängerschaft der Union strittiges und für die Mobilisierung der Grünen-Wähler eminent wichtiges Thema. Vor allem aber wiederbelebte diese entschlossene und keineswegs risikolose Reaktion den Nimbus der Kanzlerin als erfolgreiche Krisenmanagerin, den sie sich bereits bei der Banken- und Wirtschaftskrise - damals noch gemeinsam mit Finanzminister Peer Steinbrück - erworben hatte.

Den eigentlichen Wendepunkt für Merkel stellte allerdings eine andere Krise dar: die Eurokrise, die die zweite Hälfte der Regierungsperiode entscheidend prägte. Sie hatte zwar die schwarz-gelbe Regierung schon von Anfang an begleitet, spitzte sich 2011 vor allem in den überschuldeten Euro-Staaten Griechenland, Spanien und Portugal aber weiter zu. Angesichts immer höherer Zinslasten nahm das Szenario eines Staatsbankrotts besonders in Griechenland immer konkretere Züge an. Da dessen Folgen für den Euroraum insgesamt kaum kalkulierbar waren, sahen sich die anderen Mitgliedstaaten und die EZB zum Eingreifen gezwungen. Der Euro-Rettungsschirm sowie der Eurostabilitätsmechanismus (ESM) wurden installiert, im Fall Griechenlands wurde sogar ein partieller Schuldenerlass erwirkt. Aber erst die Zusicherung ihres Präsidenten Mario Draghi, dass die Europäische Zentralbank alles Notwendige unternehmen werde, um den Euro zu stützen, trug zu einer Stabilisierung der Finanzmärkte bei. Bei der Bewältigung beziehungsweise Eindämmung der Krise kam Deutschland eine herausragende Rolle zu, weil die dortige Wirtschaft im Unterschied zu den meisten anderen Euroländern boomte.

Die ökonomische Führungsposition innerhalb der EU war nie deutlicher spürbar, mit der Folge, dass Deutschland immer stärker auch in eine politische Führungsrolle gedrängt wurde. Für Merkel und ihren Finanzminister Wolfgang Schäuble bedeutete dies eine Gratwanderung, denn sie mussten die Forderung der europäischen Partner nach mehr finanzieller Unterstützung und die Befürchtungen der eigenen Bevölkerung vor einer Überforderung der Bundesrepublik unter einen Hut bringen. Dies gelang ihnen mit zunehmender Dauer der Krise - und mit kräftiger Unterstützung Draghis - immer besser. Ihre „Vorliebe (...) , auf Sicht' zu fahren und politische Prozesse weniger zu steuern als zu moderieren "16, erwies sich als die richtige Strategie, da sie der ambivalenten Haltung der Mehrheit der Deutschen durchaus entgegen kam, die zwischen Solidarität mit der Bevölkerung in den Krisenländern und Zurückweisung allzu hoher Forderungen schwankte. Die Antwort war eine „Dualität aus einem Pochen auf deutsche Interessen sowie dem Zusammenhalt der

15 Karl-Rudolf Korte, Change? Nein danke!, in: Süddeutsche.de vom 4. Dezember 2012, http:// www.sueddeutsche.de/politik/wahlkampf-von-merkel-und-obama-im-vergleich-change-neindanke-1.1540790 (Abruf 13. Januar 2014).

16 Antje Sirleschtov, Was ist vom Koalitionsvertrag geblieben?, in: Der Tagespiegel vom 6. Januar 2013, http://www.tagesspiegel.de/politik/koalitionsvertrag-was-ist-vom-geblieben/7591852.html (Abruf 13. Januar 2014). 
Eurozone “17. Am Ende waren die Bundesbürger beeindruckt von der Mischung aus Durchsetzungskraft und dem Respekt, den sich Merkel auch im Ausland mit ihrer Art der Krisenbewältigung erwarb. Dieser Reputationsgewinn machte sich in wieder deutlich steigenden Zustimmungswerten erst für die Kanzlerin, später auch für die Regierung bemerkbar. ${ }^{18}$ Bei jeder Zuspitzung der Lage wurden zwar Zweifel an dem Erfolg der Strategie deutlich - so zuletzt, als im Frühjahr 2013 auch Zypern EU-Mittel in Anspruch nehmen musste -, aber aus jeder dieser Krisen ging Merkel gestärkt hervor.

Ein weiterer positiver Nebeneffekt für die Bundesregierung stellte sich ein, weil mit jedem Bericht über die Überschuldung, die steigende Arbeitslosigkeit und die hohe Jugendarbeitslosigkeit bei den meisten europäischen Nachbarn den Deutschen vor Augen geführt wurde, wie vergleichsweise gut es ihnen ging. Klagen über zu geringe Löhne, Leiharbeit und prekäre Arbeitsplätze relativierten sich durch die viel größeren Probleme der Bürger in den meisten anderen EU-Ländern. Kurz vor der Wahl erreichte die Arbeitslosigkeit in Deutschland einen neuen Tiefststand. Mit wachsender Zuversicht, dass es gelingen werde, die Eurokrise in den Griff zu bekommen, änderte sich die Grundstimmung gegenüber der Bundesregierung. Ihre Entscheidungen fanden zunehmend den Beifall der Bevölkerung wovon allerdings nur Merkel und die Unionsparteien profitierten, die Leistungen der FDP und ihrer Minister wurden bis zum Schluss durchweg sehr kritisch bewertet.

\section{Der Wahlkampf: Positionierung der Parteien}

\subsection{CDU/CSU - Merkel als Wahlprogramm ${ }^{19}$}

CDU und CSU hatten im Wahlkampf von allen Parteien die bei weitem vorteilhafteste Ausgangslage. Die Union trat geschlossen auf, in beiden Parteien war die Führungsfrage geklärt, Merkels parteiübergreifende Führungsposition unangefochten. Die Union hatte ein klares strategisches Konzept bestehend aus drei Elementen: „Demobilisierung der Konkurrenz, Personalisierung der Kanzlerfrage, Bekämpfung des politischen Gegners als strategi-

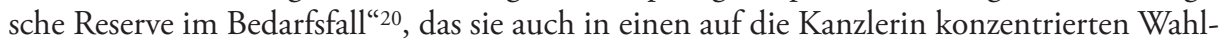
kampf umsetzen konnte. Merkel hatte das Heft des Handelns in der Hand, in zahllosen internationalen Konferenzen konnte sie sich als Krisenmanagerin medial zur Geltung bringen. Dies spiegelte sich auch in der Wahrnehmung der Bürger wider, deren 2009 noch ausgeprägte ökonomische Skepsis sich allmählich legte. Am Ende bewerteten drei von vier die wirtschaftliche Lage positiv, die eigene Situation sogar noch etwas positiver. ${ }^{21}$ Nichts lag näher, als diese Stimmung für den Grundtenor der gesamten Unions-Kampagne im Sinne

17 Karl-Rudolf Korte / Niko Switek, Regierungsbilanz: Politikwechsel und Krisenentscheidungen, in: APuZ, 63. Jg. (2013), H. 48/49, S. 3 - 9, S. 7.

18 Vgl. Infratest dimap, Wahlreport Bundestagswahl. Eine Analyse der Wahl vom 22. September 2013, Berlin 2013, S. 38.

19 Vgl. Interview mit Richard Hilmer, „Das Programm heißt Merkel“, in: tagesschau.de vom 23. Juni 2013, http://www.tagesschau.de/inland/hilmer100.html (Abruf am 13. Januar 2014).

20 Ralf Tils / Joachim Raschke, Strategie zählt. Die Bundestagswahl 2013, in: APuZ, 63. Jg. (2013), H. 48/49, S. 20 - 27, S. 21.

21 Vgl. Infratest dimap, Wahlreport Bundestagswahl. Eine Analyse der Wahl vom 22. September 2013, Berlin 2013, S. 26. 
eines „Weiter so“ nutzbar zu machen. So lautete denn auch einer ihrer Hauptslogans „Deutschland ist stark. Und soll es bleiben. “ Der Verweis auf die erfolgreiche Eindämmung der Eurokrise durfte dabei nicht fehlen: „Wachstum braucht Weitblick. Und einen stabilen Euro.“ Die Krisenstrategie Merkels, „auf Sicht zu fahren“, erwies sich wohl auch deshalb als sehr erfolgreich, weil sie der Verunsicherung der großen Mehrheit der Bürger entgegenkam, die den radikaleren Lösungsvorschlägen von Rot-Grün (Euro-Bonds) oder der AfD (Rückkehr zur DM) misstrauten.

Wie bereits 2009 verfolgte die Union auch diesmal die Strategie, einen Richtungswahlkampf so weit wie möglich zu vermeiden. Botschaften, Motive und Gestaltung waren entsprechend dezent gehalten, und erstmals fand mit der „Merkel-Raute“ ein Markenzeichen Eingang in eine politische Wahlkampagne. Deren Bewertung schwankte zwischen euphorischem Lob ${ }^{22}$ und heftiger Kritik. ${ }^{23}$ Die Personalisierung der Kanzlerfrage in den Mittelpunkt zu stellen, lag nahe, angesichts der Tatsache, dass Merkel alle Rankings als beliebteste Politikerin anführte - eine Beliebtheit, die weit über die Reihen der eigenen Anhänger hinaus reichte. Ihr gelang deshalb, was keinem ihrer Vorgänger gelungen war: die eigene Anhängerschaft maximal zu mobilisieren, ohne eine Gegenmobilisierung der Anhänger der Oppositionsparteien zu provozieren, denn eine weitere Amtsperiode Merkels löste nur bei wenigen Abwehrreaktionen aus. Auf ihrer Website www.bundeskanzlerin.de suchte sie den direkten Kontakt zu den Wählern in Form von Podcasts, Tweets des Regierungssprechers und Fotostrecken. Dabei wurde erkennbar darauf geachtet, sie zum einen in ihrer Rolle als in der Welt geachtete Politikerin, zum anderen als ganz normale Person ohne Allüren, als „Politikerin zum Anfassen“ auftreten zu lassen. In ihren Reden tauchte das Wort „gemeinsam" auffallend häufig auf, Wortwahl und Satzbau waren einfach gehalten. ${ }^{24}$ Subtil und nicht ohne Wirkung in den neuen Bundesländern, wurde diesmal auch ihre Ost-Biographie thematisiert (ihr Lieblingsfilm „Paul und Paula“). Die Fähigkeit, Nähe zu den Bürgern herzustellen, war ihr Trumpf beim TV-Duell mit dem Spitzenkandidaten der SPD. Bei den Sachthemen konnte Peer Steinbrück vielfach stärker überzeugen ${ }^{25}$, in ihrem Schlussstatement nahm Merkel aber das Publikum mit der so simplen wie genialen Aussage „Sie kennen mich (...) “ für sich ein. Unausgesprochen schwang dabei mit „(...) ich stehe für Verlässlichkeit, der Herausforderer für Risiko“.

22 „Die Merkel-Kampagne [hat] für den deutschsprachigen Raum Maßstäbe gesetzt“, so Peter Köppl, Seid professionell, in: Nicole Alexander, 14 Lehren aus dem Superwahljahr 2013, in: politik \& kommunikation, 8/2013, S. 17.

23 So etwa Heribert Prantl: „Merkel beherrscht die Kunst des Zusammenführens von Positionen auf kurios-kunstlose Weise: Sie hat keine“, ders., Große Flaute, in Süddeutsche.de vom 19. August 2013, http://www.sueddeutsche.de/politik/bundestagswahlkampf-grosse-flaute-1.1749117 (Abruf am 13. Januar 2014).

24 Vgl. Joachim Scharloth, Die Rhetorik von Angela Merkel und Peer Steinbrück im Vergleich, Polittrend: Analysen zur Bundestagswahl 2013, 19. September 2013, http://www.polittrend.de/ polittrend.de/politik/blog/? $\mathrm{p}=210 \#$ more-210 (Abruf am 13. Januar 2014).

25 Vgl. die Befunde von Frank Brettschneider, Wahlkampfkommunikation 2013: Akteure, Instrumente, Botschaften, Manuskript 11. Internationale Konferenz für politische Kommunikation, Konrad-Adenauer-Stiftung, Berlin 2013, http://www.kas.de/upload/dokumente/2013/10/ 131020_Brettschneider.pdf (Abruf am 13. Januar 2014); Ergebnisse von Infratest dimap zum TV-Duell zwischen Merkel und Steinmeier am 1. September 2013 auf tagesschau.de, http://www. tagesschau.de/inland/tv-duell-befragung100.html (Abruf am 13. Januar 2014). 
Selten gelang es auch einer Regierungspartei so erfolgreich, Wahlkampf, internationales Auftreten und Regierungshandeln aufeinander abzustimmen. Die Verabschiedung von Mindestlöhnen in zwei Branchen unmittelbar vor der Wahl dürfte dazu beigetragen haben, soziale Konflikte rechtzeitig zu entschärfen. Mit bemerkenswertem Erfolg: Zwei Wochen vor dem Wahlgang hatte erstmals seit der Vereinigung eine Mehrheit der Deutschen den Eindruck, es ginge gerecht zu im Lande ${ }^{26}$ - dem Wahlkampf der Oppositionsparteien, die auf das bis dato vorherrschende Gefühl weit verbreiteter sozialer Ungerechtigkeit setzten, war der Wind aus den Segeln genommen. ${ }^{27}$

Bleibt zu erwähnen, dass auch die CSU voll auf Merkel setzte. Selbst Horst Seehofer nahm sich im Bundestagswahlkampf weitgehend zurück, was ihm nach der Rückeroberung der absoluten Mehrheit bei der für ihn wichtigeren Landtagswahl in Bayern leicht fiel. Mit der Forderung nach einer Autobahn-Maut für Ausländer setzte er aber ein ebenso wirksames wie umstrittenes Zeichen, dass mit einer erstarkten CSU auch im Bund wieder mehr zu rechnen ist.

\subsection{FDP - Funktionspartei reicht nicht}

Der Schlüssel für das historisch beste Wahlergebnis der FDP hatte 2009 in dem Versprechen „Mehr Netto vom Brutto“ bestanden - ein Versprechen, das sie in den vier Jahren der Regierungsbeteiligung nicht ansatzweise einlösen konnte. Praktisch während der gesamten Wahlperiode mussten die Liberalen gegen die Enttäuschungserfahrungen ihrer Wähler ankämpfen. Erst versuchten sie es mit Trotz (Westerwelle in Richtung Medien: „Ihr kriegt mich nicht klein"), dann mit einem umfassenden Personalwechsel verbunden mit einem neuerlichen Versprechen („Ab heute wird geliefert“), und am Ende mit der Versicherung, Steuererhöhungen ließen sich nur mit der FDP verhindern. Zu diesem Zeitpunkt hatten die Freidemokraten aber schon fast jegliche Glaubwürdigkeit verspielt. Dies lag nicht zuletzt an einem wenig überzeugenden Personalangebot und einer am Ende ungeklärten Führungsfrage: Erstmals wurden bei der FDP Parteivorsitz und Spitzenkandidatur getrennt.

Im Wahlkampf versuchte man, an die Erfolge der Regierung anzuknüpfen („Vier gute Jahre für Deutschland“), ohne aber deutlich machen zu können, worin der spezifisch liberale Anteil an diesem Erfolg bestand. Es gab kaum ein Projekt, kaum eine Maßnahme, die sich in der Wahrnehmung der Wähler positiv mit den Liberalen verband. Selbst der erfolgreiche Widerstand der FDP-Justizministerin gegen eine allzu großzügige Datenspeicherung wurde von den Bürgern kaum wahrgenommen, obwohl das Thema durch den NSA-Skandal unmittelbar vor der Wahl hochaktuell war. Es herrschte der Eindruck vor, die FDP habe „vor der Wahl viel versprochen und fast nichts davon umgesetzt“ -83 Prozent der

26 Seit 2008 stieg der Anteil derer, die der Meinung waren, es gehe gerecht zu, um 34 auf 55 Prozent, während nur noch 40 Prozent - und damit 23 Punkte weniger - die Auffassung vertraten, es gehe ungerecht zu. Vgl. Infratest dimap, Wahlreport Bundestagswahl. Eine Analyse der Wahl vom 22. September 2013, Berlin 2013, S. 27.

27 Vgl. RalfTils / Joachim Raschke, a.a.O. (Fn. 20), S. 24: „Der sozialdemokratische Versuch, gesellschaftliche Fehlentwicklungen in den Mittelpunkt des Wahlkampfs zu rücken, ist an der Grundstimmung der Deutschen abgeprallt." 
Bundesbürger teilten diese Meinung, von den abgewanderten FDP-Wählern sogar 90 Prozent. Die Zufriedenheit mit der Regierung beschränkte sich auf Merkel und ihre CDU. ${ }^{28}$

Alarmierende Umfrageergebnisse wurden in der Hoffnung ignoriert, am Ende werde es schon gutgehen - wie zuletzt bei der Landtagswahl in Niedersachsen, als viele unionsgeneigte Wähler den gefährdeten Liberalen ihre Zweitstimme gaben in der Überzeugung, nur mit der FDP werde es für die CDU und ihren Ministerpräsidenten David McAllister reichen. Diese Hoffnung wurde erschüttert, als die Freidemokraten eine Woche vor der Wahl in Bayern mit einem Stimmenanteil von nur 3,3 Prozent den Wiedereinzug in den Landtag verpassten. Sie reagierten darauf mit einer verzweifelt anmutenden, an Unionswähler gerichteten Zweitstimmenkampagne („Wer Merkel haben will, wählt FDP“), die fehlschlug, weil sie - anders als in Niedersachsen - von der CDU deutlich zurückgewiesen wurde. ${ }^{29}$ Letztlich dürfte die Kampagne mehr geschadet als genutzt haben, weil man deutlicher kaum auf den Profilverlust und die Reduktion der FDP auf eine „Funktionspartei“ hätte hinweisen können. Liberale Positionen finden sich mittlerweile in allen Parteien, auch und besonders in der CDU. Nach deren Öffnung für "progressiv-libertäre Positionen “30 ist der Wunschpartner der FDP zum gefährlichsten Rivalen erwachsen, wie die Wählerstromanalyse bestätigt. ${ }^{31}$

\subsection{SPD - Mangel an Harmonie von Programm und Spitzenkandidat}

Nach ihrem Debakel bei der Bundestagswahl 2009 stand die SPD sowohl personell als auch programmatisch vor einem Neuanfang. Auf dem Dresdener Parteitag im November 2009 wurde Sigmar Gabriel mit großer Mehrheit zum neuen Parteivorsitzenden gewählt. In einer fulminanten Rede gelang es ihm, seine Partei moralisch wieder aufzubauen und sie gemeinsam mit einer runderneuerten Führungsspitze politisch links von der Mitte zu justieren. Diese Strategie zeitigte Erfolge: Die SPD konnte in ihrem Kernbereich soziale Gerechtigkeit an Profil gewinnen und erzielte auch wieder mehr Rückhalt in der Wählerschaft. So verliefen die meisten Landtagswahlen siegreich für die Partei. Bereits die Debatte um die Thesen von Sarrazin im Herbst 2010 machte aber deutlich, dass die SPD noch weit von alter Überzeugungskraft und Stärke entfernt blieb. Im Zuge der wieder aufflammenden Anti-Atomdebatte erwiesen sich außerdem die Grünen zunehmend als ernsthafter und vor allem in ihrem Erscheinungsbild als glaubwürdiger Konkurrent um die Führungsposition im linken Parteienspektrum - nach Fukushima rangierten sie sogar kurzfristig auf Augenhöhe mit der SPD.

28 Dies verdeutlichte ein im ARD-DeutschlandTREND August 2013 durchgeführtes Experiment: Zusätzlich zu der neutralen Frage nach der „Zufriedenheit mit der Arbeit der Bundesregierung“ (Ergebnis: 52 Prozent sehr zufrieden/zufrieden) wurde im Split-Verfahren auch nach der mit der „(...) Bundesregierung unter Führung von Angela Merkel“ (56 Prozent) sowie nach der mit „der Bundesregierung aus CDU/CSU und FDP“ gefragt. Die bloße Erwähnung der FDP ließ die Werte auf 38 Prozent sinken.

29 Die Kampagne wurde von Merkel in einem in der Wahlwoche an fünf Millionen Haushalte verteilten Schreiben gekontert: „Wenn Sie möchten, dass ich weiter als ihre Kanzlerin arbeiten kann, dann (...) geben Sie beide Stimmen der CDU.“

30 Karl-Rudolf Korte / Niko Switek, a.a.O. (Fn. 17), S. 6.

31 Siehe auch Kapitel 4.1. 
Die bereits 2011 einsetzende Debatte um die Kanzlerkandidatur sicherte den Sozialdemokraten zwar eine ständige Medienpräsenz; sie zeigte aber auch, dass die Führungsfrage keineswegs gelöst war. Gehandelt wurden drei Kandidaten, wovon Gabriel zwar formell den ersten Zugriff hatte, Frank-Walter Steinmeier aber den größeren Rückhalt in der Fraktion und Peer Steinbrück den höchsten Zuspruch in der Wählerschaft. Die Entscheidung war eigentlich für Ende 2012 angesetzt; als aber Steinmeier unabgestimmt Ende September 2012 seinen Verzicht erklärte, kürte Gabriel umgehend Steinbrück zum Kanzlerkandidaten der SPD. Diese Entscheidung fiel nicht zuletzt in der Hoffnung, dass der ehemalige Finanzminister am ehesten Wähler der Mitte ansprechen könne. Das wäre angesichts des starken Rückhalts der Kanzlerin bei den bürgerlichen Wählern für sich genommen schon schwierig gewesen, angesichts der überstürzten Nominierung - Steinbrück verfügte zu diesem Zeitpunkt weder über eine Mannschaft noch über ein Konzept oder eine Strategie war dies eine überambitionierte Zielsetzung.

Schon wenige Tage nach seiner Nominierung geriet Steinbrück zudem in die Defensive: Der Vorwurf, Einkünfte für Auftritte als Redner nicht richtig deklariert zu haben, erwies sich zwar als haltlos, gleichwohl musste er „mangelndes Fingerspitzengefühl“32 bei mancher Honorarforderung einräumen. Es folgten weitere verunglückte Interviewäußerungen, die zum Teil von der Presse hochgespielt wurden („Pinot-Grigio-Affäre“33), teilweise aber auch Zweifel an seiner Glaubwürdigkeit in der eigenen Wählerschaft nährten. Dazu zählte vor allem seine Kritik an der zu geringen Höhe des Kanzlergehaltes. Die wahlstrategische Ausrichtung der SPD als der Partei der sozialen Gerechtigkeit und das Kandidatenimage drohten in Widerspruch zueinander zu geraten. Die Positionierung des Spitzenkandidaten als Politiker, der pointiert argumentiert, „Klartext redet“ - gleichsam als Gegenmodell zum „erklärungsarmen Pragmatismus“34 der Kanzlerin -, erwies sich gelegentlich als Risiko. Die Titulierung zweier führender italienischer Politiker als „Clowns“ brachte ihm sogar einen Protest des italienischen Staatspräsidenten ein. Und als er Merkel in einem Gespräch mit dem Tagesspiegel einen Mangel an Leidenschaft für Europa vorwarf und diesen mit ihrer DDR-Sozialisation in Zusammenhang brachte, sorgte er bei vielen Ostdeutschen für Empörung. Letztlich trug all dies dazu bei, Merkel in noch besserem Licht erscheinen zu lassen. Steinbrück verlor schon kurz nach seiner Nominierung, die SPD mit zeitlicher Verzögerung deutlich an Zustimmung.

Der Wahlkampf begann überstürzt, wollte bis zum Sommer nicht wirklich in Schwung kommen und setzte sich mit unglücklichen Personalentscheidungen sowie bisweilen mangelnder Abstimmung zwischen Spitzenkandidat, Wahlkampfführung und Parteispitze fort. Die SPD verharrte in den Umfragen um die 25 Prozent und drohte sogar noch weiter abzurutschen, da Rot-Grün als Alternative zur amtierenden schwarz-gelben Koalition zunehmend an Strahlkraft verlor und das zentrale Thema soziale Gerechtigkeit nicht zündete. Einzig beim TV-Duell mit Steinbrück in Hochform war ansatzweise erkennbar, was mit einem gelungenen Wahlkampf hätte erreicht werden können. In direkter Auseinandersetzung

32 Steinbrück in der Defensive, in: Handelsblatt vom 11. November 2012, http://www.handelsblatt. $\mathrm{com} /$ politik/deutschland/rednerhonorare-steinbrueck-in-der-defensive/7375974.html (Abruf am 13. Januar 2014).

33 Steinbrück hatte auf einer Diskussionsveranstaltung gesagt, er kaufe keinen Pinot Grigio für unter 5 Euro die Flasche.

34 Karl-Rudolf Korte / Niko Switek, a.a.O. (Fn. 17), S. 8. 
mit Merkel konnte er vor allem bei den für die SPD wichtigen sozialen Themen wie Mindestlohn, Alterssicherung und Pflege überzeugen, was ihm sogar einen knappen „Punktsieg“ einbrachte. ${ }^{35}$ Ein kurzfristiger Aufwärtstrend für die SPD wurde allerdings durch Steinbrücks Stinkefinger-Foto auf der Titelseite des SZ-Magazins konterkariert, das in der Woche vor der Wahl noch einmal für Schlagzeilen sorgte. ${ }^{36}$

\subsection{Grüne - Wahlkampf jenseits des Markenkerns ${ }^{37}$}

Die Grünen starteten mit hohen Erwartungen in den Wahlkampf; fraglich schien nur, wie hoch ihre Zugewinne ausfallen würden. Dass sie am Ende sogar deutlich an Stimmen verloren, lag sicher nicht nur, aber auch an ihrem Wahlkampf. Darauf mussten die Grünen lange Zeit keinen Gedanken verschwenden, weil es für sie ohne großes Zutun rasant bergauf ging. Die Partei profitierte von einer von ihren Kernthemen dominierten Agenda: Bürgerbeteiligung, Atomkraft, Integrationspolitik. Und im Frühjahr lieferte die Atomkatastrophe in Fukushima den empirischen Beleg für die Berechtigung grüner Skepsis gegenüber Atomkraftwerken. In den Umfragen stiegen die Grünen bis nahe an die 25-Prozent-Marke. Der von Merkel als Konsequenz aus Fukushima beschlossene Ausstieg aus der Kernkraftnutzung und die Ausrufung der Energiewende markierten den entscheidenden Wendepunkt. Schon früh zeichnete sich ab, dass den Grünen das Thema Atomenergie als wichtiges Mobilisierungsinstrument fehlen würde. Tatsächlich fiel der Wählerrückhalt bis März 2012 mit 13 Prozent wieder fast auf den Ausgangswert der letzten Bundestagswahl.

Der Mitgliederentscheid über die Spitzenkandidaten brachte ihnen wieder mehr mediale Aufmerksamkeit und auch mehr Zuspruch bei den Wählern. Bei der Befragung setzten sich - erwartungsgemäß - Jürgen Trittin sowie - überraschend - Katrin Göring-Eckardt durch. Letztere schien den Mitgliedern eher geeignet als die bekannteren Renate Künast und Claudia Roth, über die grüne Stammklientel hinaus Wähler anzusprechen. Auf ihrem Parteitag im Februar 2013 verabschiedeten sie ein über 300 Seiten umfassendes Wahlprogramm, in dem alle klassischen grünen Themen aufgegriffen wurden. Dominiert wurde aber die Diskussion auf dem Parteitag ${ }^{38}$ und später auch die Medienberichterstattung von dem unter dem Titel „Besser haushalten“ abgehandelten Thema „Steuern“. Gefordert wurde dort eine Erhöhung der Einkommens- und der Erbschaftssteuer, die Wiedereinführung der Vermögenssteuer sowie die Abschaffung des Ehegattensplittings. ${ }^{39}$ Anstatt Umweltpolitik und Energiewende beherrschte die Forderung nach mehr Verteilungsgerechtigkeit den

35 Vgl. Frank Brettschneider, a.a.O. (Fn. 25); Infratest dimap, a.a.O. (Fn. 25).

36 Das Bild entstand im „Sagen Sie jetzt nichts“-Interview auf die Frage: „Pannen-Peer, ProblemPeer, Peerlusconi - um nette Spitznamen müssen Sie sich keine Sorgen machen, oder?“, in: SZ-Magazin vom 13. September 2013.

37 Vgl. Hans-Hermann Langguth, Unterschätzt den Gegner nicht, in: Nicole Alexander, a.a.O. (Fn. 22), S. 16.

38 Die FAZ konstatierte erstaunt: „Die Grünen diskutieren auf ihrem Parteitag mehr über Steuern als über die Umwelt", Johannes Leithäuser, Ein Rucksack voller Übermut, in: FAZ vom 28. April 2013, http://www.faz.net/aktuell/politik/bundestagswahl/parteien-und-kandidaten/gruenen-parteitag-ein-rucksack-voller-uebermut-12165792.html (Abruf am 13. Januar 2014).

39 Das Splitting sollte ersetzt werden durch „eine Individualbesteuerung mit übertragbarem Existenzminimum". 
gesamten Wahlkampf der Grünen. Ihnen zugetane bürgerliche Wähler wurden - anders als etwa bei der Landtagswahl in Baden-Württemberg - kaum mehr angesprochen. Selbst die „grüne Mittelschicht“, die für einen finanziellen Beitrag zur Bekämpfung des Klimawandels oder zur Einführung erneuerbarer Energien zu überzeugen gewesen wäre, kam über die „schwer absehbare Kumulation von Steuern und die Grauzone der Betroffenheit“ ${ }^{40}$ ins Grübeln. Mit der Forderung nach einem „Veggie-Day“ lieferte man der politischen Konkurrenz einen weiteren Anlass, die Grünen als „Bevormundungspartei“ zu bezeichnen. ${ }^{41}$ Die unmittelbar vor der Wahl losgetretene Debatte über pädophile Strömungen der Partei in den 1980er Jahren beschädigte ihre wertvollste Ressource: Glaubwürdigkeit. Diese Entwicklungen sorgten für tiefe Verunsicherung und veranlassten auch Stammwähler, sich von den Grünen abzuwenden. ${ }^{42}$

\subsection{Linke - Im Schatten der Anderen: Gysis erfolgreiches Gefecht}

Ganz anders verlief der Wahlkampf bei der Linken. Dort hatte man sich auf deutliche Verluste eingestellt. 2009 noch zweistellig in den Bundestag eingezogen, folgte bald die Trendwende. Nach dem angekündigten Rückzug von Oskar Lafontaine aus der Bundespolitik verzichtete auch Lothar Bisky auf eine erneute Kandidatur für den Parteivorsitz. Das neue Führungsduo Gesine Lötzsch und Klaus Ernst konnte an die Erfolge ihrer Vorgänger nicht anknüpfen und die innerparteilichen Diskussionen über die strategische Ausrichtung der Partei insbesondere hinsichtlich der Frage Regierungsbeteiligung oder Fundamentalopposition nicht bändigen. Die Landtagswahlen 2011 in Baden-Württemberg und RheinlandPfalz markierten ein abruptes Ende der „Westexpansion“ der Linken. In beiden Ländern kam die Partei nicht einmal in die Nähe der Fünf-Prozent-Hürde. In der ersten Jahreshälfte 2012 geriet sie zusätzlich unter Druck, weil die Piraten einen erheblichen Teil des linken Protestpotentials aufsogen. Nach den herben Wahlschlappen in Nordrhein-Westfalen und Schleswig-Holstein, als die Linke aus beiden Landtagen herausfiel, und den heftigen Auseinandersetzungen um ein mögliches Comeback von Oskar Lafontaine an der Parteispitze landete die Partei in der Infratest-dimap-Sonntagsfrage erstmalig bei nur noch fünf Prozent. Der Wiedereinzug in den Bundestag war damit ernsthaft in Frage gestellt. Dem im Juni 2012 neu installierten Führungsduo Katja Kipping und Bernd Riexinger gelang es jedoch, die innerparteilichen Diskussionen etwas zu beruhigen und die Linke aus den dauernden Negativschlagzeilen herauszuführen. Infolgedessen stabilisierte sie sich auch demoskopisch wieder - zwar nur leicht, aber stabil - über der Fünf-Prozent-Hürde.

Auch das Wahljahr begann für die Linken nicht harmonisch. Die Auseinandersetzungen um die Spitzenkandidatur und die damit verbundenen Richtungsentscheidungen mündeten schließlich in ein achtköpfiges Spitzenkandidatenteam. Faktisch kristallisierte sich aber

40 Vgl. Joachim Raschke / RalfTils, a.a.O. (Fn. 20), S. 26.

41 Philipp Rösler bezichtigt sie der "Spießbürgerlichkeit“ und des „Tugendwahns“, vgl. Rösler findet Grüne spießig, in: taz.de vom 4. Mai 2013, http://www.taz.de/!115726/ (Abruf am 13. Januar 2014).

42 Die Haltequote - der Anteil der Wähler, die in Umfragen angeben, derselben Partei die Stimmen geben zu wollen wie vier Jahre zuvor - sank bei den Grünen in den letzten Wochen vor der Wahl von ehemals knapp 80 auf unter 50 Prozent. 
im Laufe des Wahlkampfes Gregor Gysi als eigentliche Nummer eins heraus. Lediglich Sarah Wagenknecht konnte noch ein wenig mediale Aufmerksamkeit für sich gewinnen. Inhaltlich konzentrierte sich die Linke - anders als die Grünen - auf ihre traditionellen Kernkompetenzen: Soziale Gerechtigkeit (unter anderem 10-Euro-Mindestlohn, Millionärssteuer und Abschaffung von Hartz-IV), Pazifismus (Rückzug aus Afghanistan und keine militärische Einmischung in Syrien) und Ostdeutschland. Von Vorteil für die Partei war, dass eine Regierungsbeteiligung dank des kategorischen Ausschlusses von Rot-Rot-Grün durch Steinbrück und Gabriel im Wahlkampf nicht zur Debatte stand. Dies verhinderte zum einen ein erneutes Aufleben der innerparteilichen Flügelkämpfe über die langfristige Ausrichtung. Zum anderen konnten die sozial-, steuer- und außenpolitischen Forderungen ohne Rücksicht auf eine später nötige Kompromissfindung auf klare Botschaften zugespitzt und transportiert werden, wobei sie zum Ende des Wahlkampfs auch von Schwächen bei SPD und Grünen profitierte.

\subsection{AfD und Piraten - Ein-Themen-Parteien im Auf- und Abschwung}

Die erst im Februar 2013 gegründete „Alternative für Deutschland“ (AfD) setzte ihre Hoffnungen auf den Einzug in den Bundestag in erster Linie auf das Thema Euro. Mit der Forderung nach einer Auflösung des gemeinsamen Währungsraumes und des Stopps der Eurorettungsprogramme versuchte die AfD, das durchaus vorhandene Unbehagen bei vielen Bürgern über die Eurorettungspolitik zu nutzen. Hauptadressat ihres Wahlkampfes waren von der Krisenpolitik der Kanzlerin nicht überzeugte bürgerlich-konservative Wähler. Außerdem setzte sie immer wieder auf rechtspopulistische Themen und Argumentationsmuster, von der vermeintlichen direkten Einwanderung in die Sozialsysteme bis hin zu Verschwörungstheorien über die „entartete“ Demokratie. Auch wenn beispielsweise explizit die Stimmen ehemaliger NPD-Wähler begrüßt wurden, bemühte sich die AfD um einen möglichst seriösen Anstrich und vertrat einen „Saubermann-Populismus“, bei dem sich „Ressentiments hinter der Fassade feiner Herren in Nadelstreifen mit Professorentiteln “43 verbergen konnten. Von Vorteil war für die AfD auch, dass sie mit Bernd Lucke einen innerparteilich unumstrittenen Spitzenkandidaten hatte, der fast alle mediale Aufmerksamkeit auf sich bündeln konnte.

Ganz anders die Piraten: Nach ihrem Überraschungserfolg bei der Abgeordnetenhauswahl in Berlin im Herbst 2011 surften sie in der ersten Jahreshälfte 2012 getragen von einem Medienhype und einer Protestwelle in drei weitere Landtage und zu zweistelligen bundesweiten Ergebnissen in der Sonntagsfrage. Mangelnde programmatische Substanz, vor allem aber nicht endende, stets öffentlich ausgetragene heftige innerparteiliche Querelen verbunden mit einem hohen Verschleiß an Führungskräften ließen den Stern der Piraten schnell wieder sinken. Bereits im Oktober 2012 lagen sie das letzte Mal in der bundesweiten Sonntagsfrage bei fünf Prozent. Im Wahlkampf spielten sie dann eine kaum noch wahrnehmbare Rolle, obwohl mit den Enthüllungen von Edward Snowden und der NSA-Affäre ein zumindest vordergründig piratenaffines Thema im Wahlkampf hohe Wellen schlug. 


\section{Das Wahlergebnis im Detail ${ }^{44}$}

\subsection{Wählerwanderung}

Die starken Zugewinne der Union sind fast ohne Beispiel in der Geschichte der Bundestagwahlen. Prozentual hat keine Partei nach 1953 bei einer Wahl stärker zulegen können. Auch in absoluten Zahlen gab es nach 1990 keinen größeren Zugewinn für eine Partei beziehungsweise für CDU und CSU gemeinsam: 3,51 Millionen Wähler konnten die beiden Unionsparteien mehr von sich überzeugen als noch 2009. Wie die Wählerwanderungsbilanz von Infratest dimap ${ }^{45}$ zeigt, konnten die Unionsparteien dabei von allen anderen Parteien Wähler gewinnen. Am stärksten war der Zustrom von der FDP. Musste die Union sowohl 2005 als auch 2009 noch jeweils mehr als eine Million Wähler an die Liberalen abgeben, wechselten 2013 2,11 Millionen Wähler von der FDP zur Union. Zweite Quelle der starken Zuwächse von CDU/CSU war die Mobilisierung ehemaliger Nichtwähler (+1,13 Millionen). Die Attraktivität der Union unter Führung Merkels wirkte aber auch über die Lagergrenzen hinweg. Die Zugewinne aus dem linken Lager summieren sich auf immerhin 750.000 Wähler (von den Grünen 420.000, von der SPD 210.000 und von der Linkspartei 120.000). Abgeben mussten CDU/CSU lediglich 290.000 Wähler an die erstmals angetretene AfD. Zudem litten sie nach wie vor unter der Überalterung ihrer Wählerschaft. Weil im Laufe der Wahlperiode mehr ehemalige Unions-Wähler verstarben, als bei den Erstwählern gewonnen werden konnten, bleibt per Saldo ein Verlust von 490.000 Wählern im Generationenwechsel.

Die SPD konnte ihren nach 1998 gestarteten dramatischen Abwärtstrend stoppen und auch auf Ebene der absoluten Zahlen wieder zulegen. Angesichts von über zehn Millionen Wählern, die zwischen 1998 und 2009 der SPD den Rücken gekehrt haben, fielen die Zugewinne (+1,26 Millionen) allerdings eher bescheiden aus. Die Zugewinne kamen dabei vor allem von den kleinen Bundestagsparteien, von den Grünen 550.000 Wähler, von den Liberalen 530.000 und von der Linken 370.000. Anders als von den Wahlkampfstrategen der SPD geplant, konnten weder von der Union Wähler gewonnen werden (-210.000), noch konnte die eigene Mobilisierungsschwäche behoben werden. Zwar wurden 360.000 ehemalige Nichtwähler mobilisiert; der große Einbruch bei der Wahl 2009, als über zwei Millionen SPD-Wähler zuhause blieben, wurde aber nicht annähernd wieder ausgeglichen.

Größter Verlierer 2013 sind ohne Zweifel die Liberalen. Sie erzielten nicht einmal mehr ein Drittel ihrer Stimmen von 2009 und mussten einen Verlust von 4,23 Millionen Wählern hinnehmen. Die Verluste gingen dabei in alle Richtungen. Allein die Hälfte (2,11 Millionen) waren Wechsler zum bisherigen Koalitionspartner Union. Etwa jeweils eine hal-

44 Sofern nicht anders angegeben vgl. zu allen Zahlenwerten in diesem Kapitel Infratest dimap, Wahlreport Bundestagswahl. Eine Analyse der Wahl vom 22. September 2013, Berlin 2013; Infratest dimap, ARD-DeutschlandTREND September 2013, Berlin 2013.

$45 \mathrm{Zu}$ den methodischen Grundlagen vgl. Richard Hilmer / Michael Kunert, Wählerwanderung: Das Modell von Infratest dimap, in: Jürgen W. Falter / Oscar W. Gabriel / Bernhard Weßels (Hrsg.), Wahlen und Wähler. Analysen aus Anlass der Bundestagswahl 2002, Wiesbaden 2005, S. 134 156. Für eine summarische Betrachtung der Wählerwanderungen 1998 bis 2009 vgl. Stefan Merz / Jürgen Hofrichter, Wähler auf der Flucht: die Wählerwanderung zur Bundestagwahl 2009, in: Bernhard Weßels / Harald Schoen / Oscar W. Gabriel (Hrsg.), Wahlen und Wähler. Analysen aus Anlass der Bundestagswahl 2009, Wiesbaden 2013, S. 97 - 117. 
be Million Wähler gingen der FDP im Austausch mit SPD (-530.000) und AfD (-430.000) sowie durch Abwanderung zu den Nichtwählern (-460.000) verloren.

Nachdem die Linke bei den letzten beiden Bundestagswahlen vor allem auf Kosten der SPD Stimmen gewinnen konnte, musste sie 2013 deutliche Verluste hinnehmen (1,4 Millionen). Diese verteilten sich dabei in erster Linie und fast zu gleichen Teilen auf die SPD (-370.000), auf die AfD (-340.000) und auf die Nichtwähler (-320.000).

Die Grünen verloren in absoluten Zahlen 950.000 Stimmen. Erstmals nach 1998 haben sie Wähler an die SPD abgegeben (-550.000). Ebenfalls ungewöhnlich waren die starken Verluste an die Union (-420.000).

Die neu gegründete AfD erhielt 2,06 Millionen Zweitstimmen. Für eine Protestpartei typisch, gewann sie Wähler unterschiedlichster Couleur. An erster Stelle steht ein Zustrom von den Liberalen (+430.000). Es folgen Zugewinne von der Linken (+340.000), von der Union (+290.000) und die Mobilisierung ehemaliger Nichtwähler $(+210.000)$. Die bei neuen Protestparteien üblichen Zugewinne bei den zahlreichen Kleinstparteien summierten sich auf 410.000. Auf ehemalige SPD- und Grünen-Wähler wirkte die AfD dagegen vergleichsweise wenig attraktiv (180.000 von der SPD, 90.000 von den Grünen).

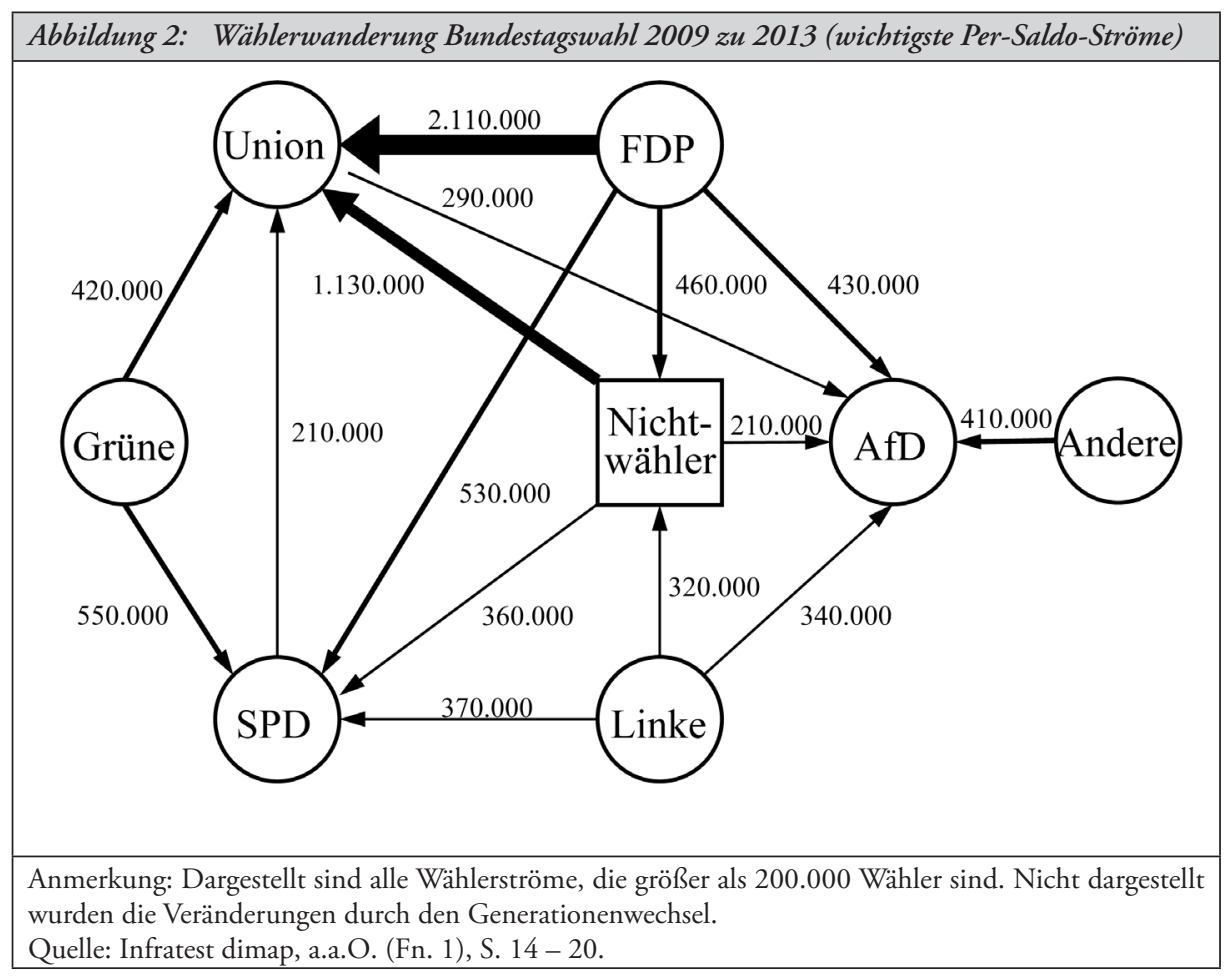

Die Wanderungsströme unterscheiden sich nicht fundamental zwischen West- und Ostdeutschland. Auffällig ist, dass die großen Bewegungen weg von der FDP und in geringerem Umfang weg von den Grünen insbesondere im Westen stattgefunden haben. Sowohl die Zugewinne der Union von der FDP und den Grünen als auch die der SPD von der FDP, den Grünen und der Linken sind im Westen überproportional ausgeprägt. Dem- 
gegenüber konnte die Union ehemalige Nichtwähler im Osten deutlich stärker mobilisieren als im Westen.

\subsection{Wahlverhalten nach Regionen}

Die Unterschiede im Wahlverhalten zwischen alten und neuen Bundesländern haben sich bei dieser Wahl insgesamt leicht verringert. Dank überdurchschnittlicher Zugewinne der Union im Osten hat sich bei ihr das West-Ost-Gefälle abgeflacht. Ähnlich ist der Fall bei FDP, Grünen und Linken, die allesamt jeweils in jenem Landesteil überproportional stark verloren haben, in dem sie traditionell stärker abschneiden. Damit haben auch bei diesen Parteien die Ost-West-Unterschiede abgenommen. Gegenläufig ist dagegen die Entwicklung bei der SPD. Während die Sozialdemokraten im Westen deutlich zulegen konnten, stagnierten sie im Ostteil des Landes. Größer wurden die Unterschiede auch bei den sonstigen Parteien. Durch das starke Ergebnis der AfD im Osten hat sich das Ost-West-Gefälle bei den Protestparteien und Kleinparteien deutlich verstärkt.

Die Union konnte flächendeckend ihre Stimmenanteile ausbauen, verzeichnete sowohl auf Bundesland- als auch auf Wahlkreisebene durchweg Zugewinne. Am wenigsten zulegen konnte sie in den Stadtstaaten Hamburg $(+4,3$ Prozentpunkte), Bremen $(+5,4)$ und Berlin $(+5,6)$. Hier schnitt sie im Bundesländervergleich auch am schlechtesten ab (Berlin 28,5, Bremen 29,3 und Hamburg 32,1 Prozent). Ebenfalls unterdurchschnittlich blieb der Zuwachs der CSU in Bayern (+6,7), auch wenn sie mit 49,3 Prozent nach wie vor der erfolgreichste Landesverband der Union ist. Dank zweistelliger Zugewinne (+11,2 Prozentpunkten) ist die baden-württembergische CDU mit 45,7 Prozent der CSU aber dicht auf den Fersen. Ebenfalls stark überdurchschnittlich fielen die Ergebnisse für die CDU in Brandenburg $(+11,2)$, Sachsen-Anhalt $(+11,1)$ und in Mecklenburg-Vorpommern $(+9,4)$ aus. Am besten schneidet die Union wie gewohnt im katholisch-ländlichen Raum ab. Spitzenreiter ist (wie schon 2009) der Wahlkreis 32 Cloppenburg-Vechta mit einem CDU-Ergebnis von 63,2 Prozent. Angeführt von Rottal-Inn finden sich sieben bayerische Wahlkreise in der Top-Ten-Liste der Union. Hinzu kommen dank zweistelliger Zuwächse der baden-württembergische Wahlkreis 292 Biberach sowie der niedersächsische Wahlkreis 31 Mittelems. Die größten Zugewinne erzielte die Union im Wahlkreis 295 Zollernalb-Sigmaringen mit einem Plus von 13,8 Prozentpunkten, gefolgt von sechs weiteren württembergischen Wahlkreisen mit Gewinnen von 13 Prozentpunkten und mehr. Diese deutlichen Zugewinne gehen einher mit ebenso starken Verlusten der FDP.

Auch wenn die Union flächendeckend zulegen konnte, bleibt sie nach wie vor in den Großstädten vergleichsweise schwach. Die 20 schlechtesten Ergebnisse liegen durchweg in Großstädten. Angeführt vom Wahlkreis 83 Berlin-Friedrichshain-Kreuzberg-Prenzlauer Berg-Ost (15,4 Prozent) zählen dazu sechs weitere Berliner, acht Ruhrgebiets- und fünf Wahlkreise aus Hamburg, Bremen und Hannover. Mit Ausnahme von Stuttgart (+9,9 Prozentpunkte) blieben die Zuwächse in allen Großstädten über 400.000 Einwohner deutlich unter dem Zugewinn bundesweit.

Der Nordwesten Deutschlands ist wie bei den vorangegangen Bundestagswahlen das günstigste Terrain für die Sozialdemokraten. Ihr bestes Ergebnis erzielte die SPD in Bremen (35,6 Prozent), es folgen die Bundesländer Niedersachsen $(33,1)$, Hamburg $(32,4)$, Nordrhein-Westfalen $(31,9)$ und Schleswig-Holstein $(31,5)$. Im Süden und Osten bleibt die 
SPD dagegen weit hinter ihrem Bundesergebnis zurück. In den ostdeutschen Flächenländern landet sie (mit Ausnahme Brandenburgs) weit unter der 20-Prozent-Marke und hinter CDU und Linke nur auf dem dritten Platz. Diese Bundesland-Unterschiede haben sich gegenüber 2009 deutlich verschärft. Zum einen konnten die Sozialdemokraten im Osten kaum zulegen - in Brandenburg (-2,0 Prozentpunkte) und in Thüringen $(-1,5)$ schnitten sie sogar schlechter ab als zuletzt. Zum anderen liegen die stärksten Zuwächse in den nordwestdeutschen Hochburgen (Bremen $+5,3$, Hamburg $+5,0$, Schleswig-Holstein $+4,8$ ). Lediglich das Saarland mit einem Plus von 6,3 Prozentpunkten durchbricht dieses Muster. Die besten SPD-Wahlkreise liegen traditionell fast ausschließlich im Ruhrgebiet. Hinzu kommen einige wenige von Bergbau und der Werftindustrie geprägte niedersächsische Wahlkreise. Allerdings kommt die SPD auch in ihren Stammrevieren nicht mehr an die 50-Prozent-Marke heran. Spitzenreiter ist mit 44,0 Prozent der Wahlkreis 123 Gelsenkirchen. Neun der zehn Wahlkreise mit dem schlechtesten SPD-Ergebnis liegen in Sachsen. Im Wahlkreis 158 Sächsische Schweiz-Osterzgebirge schafft es die SPD lediglich auf 10,9 Prozent. Im Westen finden sich die schwächsten SPD-Ergebnisse wie gewohnt in ländlichen Teilen Bayerns.

Der dramatische Einbruch der FDP zieht sich durch die gesamte Bundesrepublik. In nahezu allen Bundesländern schrumpfte der Stimmenanteil auf etwa ein Drittel des Anteils von 2009. Am stärksten waren die Verluste in den Bundesländern, in denen die FDP 2009 ihre besten Ergebnisse erzielte: Baden-Württemberg (-12,6 Prozentpunkte), RheinlandPfalz $(-11,1)$ und Hessen $(-11,0)$. Überall fielen die Liberalen hinter die Grünen zurück. Lediglich in Bayern, Baden-Württemberg und Rheinland-Pfalz konnten sie sich noch vor der Linken platzieren. Noch am besten schnitten sie mit 6,2 Prozent in ihrem Stammland Baden-Württemberg ab. Das Ergebnis im Westen hätte mit 5,2 Prozent noch knapp für einen Einzug in den Bundestag gereicht. Im Osten schafften die Liberalen aber nur 2,9 Prozent, so dass sie insgesamt unter der Mandatsschwelle blieben. In vier der sechs ostdeutschen Länder landeten die Liberalen sogar noch hinter der NPD auf dem siebten Platz im Parteienwettbewerb. Die starken Verluste der FDP ziehen sich auch durch alle Wahlkreise. Die zehn Wahlkreise mit den größten Verlusten liegen durchweg in Baden-Württemberg. Spitzenreiter ist Zollernalb-Sigmaringen mit einem Minus von 15,8 Prozentpunkten. Während die Liberalen 2009 noch in 270 der 299 Wahlkreisen ein zweistelliges Ergebnis erzielen konnten, schafften sie das 2013 in keinem einzigen Wahlkreis mehr. Ihr bestes Ergebnis erzielten sie im Wahlkreis 106 Düsseldorf I (9,2 Prozent).

Auch die Linke muss nahezu flächendeckend Verluste verkraften. Am höchsten sind diese in der Lafontaine-Heimat Saarland (-11,2 Prozentpunkte), durchweg überdurchschnittlich in den ostdeutschen Flächenländern, allerdings kann sich die Linkspartei hier immer noch über der 20-Prozent-Marke halten. Im Westen insgesamt reichte es für den Sprung über die Fünf-Prozent-Hürde (5,4 Prozent); lediglich in Baden-Württemberg $(4,8)$ und Bayern $(3,8)$ schaffte sie die Mandatsschwelle nicht. Am geringsten sind die Verluste in Berlin (-1,7 Prozentpunkte). Hier - im Bundesland des Spitzenkandidaten Gysi - liegen auch die drei besten Wahlkreise der Linken (Berlin-Lichtenberg 34,6, Berlin-Marzahn-Hellersdorf 32,9 und Berlin-Treptow-Köpenick 29,5 Prozent) und die einzigen vier (Wahlkreise 80 bis 83), in denen die Linke keine Stimmen verlor. Zweistellige Verluste gab es lediglich in den vier Wahlkreisen des Saarlandes, am höchsten fielen sie in Saarbrücken aus (-12,3).

Die Grünen erzielen ihre besten Ergebnisse traditionell in den Stadtstaaten, so auch trotz überdurchschnittlichen Rückgangs - 2013 in Hamburg (12,7 Prozent), Berlin (12,3) 
und Bremen (12,1). Zweistellig schneiden sie auch im grün-rot regierten Baden-Württemberg $(11,0)$ ab. Auch wenn die Grünen in ihren Hochburgen überdurchschnittlich stark abgeben mussten, schneiden sie nach wie vor am besten in den Stadtstaaten, den großen westdeutschen Metropolen und in prosperierenden Dienstleistungs- und Universitätsstädten ab. Spitzenreiter unter den Wahlkreisen ist Berlin-Friedrichshain-Kreuzberg-Prenzlauer Berg-Ost (20,8). Es folgen die Wahlkreise 281 Freiburg $(19,8)$ und 258 Stuttgart I $(17,5)$. Verluste mussten die Grünen in allen Bundesländern und in allen Wahlkreisen hinnehmen, am wenigsten noch im Saarland (-1,1 Prozentpunkte) und in den ostdeutschen Flächenländern $(-1,1$ bis $-1,8)$. Allerdings fiel der Stimmenanteil der Grünen damit im Osten überall (mit Ausnahme Berlins) wieder unter die Fünf-Prozent-Hürde. Ihr schlechtestes Ergebnis erzielten sie in Sachsen-Anhalt mit 4,0 Prozent.

Die erstmalig angetretene AfD verfehlte zwar den Einzug in den Bundestag knapp, konnte aber in immerhin sieben Bundesländern die Fünf-Prozent-Hürde überspringen. Am besten schnitt sie in Sachsen (6,8 Prozent), Thüringen $(6,2)$ und Brandenburg $(6,0)$, am schwächsten in Bremen (3,7), Niedersachsen (3,7) und Nordrhein-Westfalen (3,9) ab. Die Wahlkreisergebnisse werden vom Wahlkreis 157 Görlitz $(8,2)$ angeführt, gefolgt von einer ganzen Reihe an sächsischen und thüringischen Wahlkreisen. Im Westen konnte die AfD die meisten Stimmen im Wahlkreis 279 Pforzheim $(7,2)$ sowie im Frankfurter Umland (WK 176 Hochtaunus und WK 181 Main-Taunus jeweils 6,9) holen.

Die ebenfalls an der Fünf-Prozent-Hürde gescheiterten Piraten erzielten ihr bestes Landesergebnis in Berlin (3,6 Prozent). Hier liegt auch der einzige Wahlkreis, in dem sie über die Mandatsschwelle kamen (Wahlkreis 83 Berlin-Friedrichshain-Prenzlauer Berg Ost mit 5,8 Prozent). In lediglich 21 ausnahmslos großstädtischen Wahlkreisen gewannen die Piraten mehr als drei Prozent.

Die Wahlbeteiligung stieg insgesamt nur leicht (+0,7 Prozentpunkte). Im Westen blieb sie fast unverändert $(+0,2)$. In sechs Bundesländern nahm sie sogar ab, am stärksten in Bayern $(-1,6)$ und Bremen $(-1,5)$. Mit 74,3 Prozent die höchste Wahlbeteiligung und mit 1,8 Prozentpunkten den höchsten Anstieg im Westen verzeichnete Baden-Württemberg. Im Osten lag die Beteiligung insgesamt deutlich niedriger als im Westen. Schlusslicht ist - wie schon bei früheren Bundestagswahlen - Sachsen-Anhalt mit 62,1 Prozent. Auch in den anderen ostdeutschen Flächenländern blieb die Wahlbeteiligung unter 70 Prozent. Allerdings legte die Wahlbeteiligung im Osten deutlich stärker zu (+2,7 Prozentpunkte) als im Westen, so dass sich das traditionelle West-Ost-Gefälle bei der Wahlbeteiligung merklich abgeflacht hat.

Der gegenüber 2009 noch einmal gestiegene Vorsprung der Union vor der SPD führte zu einer noch größeren Dominanz der Union bei den Wahlkreisgewinnern. Von den 299 Direktmandaten gingen 236 an Kandidaten von CDU oder CSU, 18 mehr als 2009. Wie schon 2009 in Bayern, in Sachsen und im Saarland konnte die Union dieses Mal auch in Baden-Württemberg, Mecklenburg-Vorpommern, Sachsen-Anhalt und Thüringen sämtliche Wahlkreise gewinnen. Kandidaten der SPD waren in 58 Wahlkreisen erfolgreich, sechs weniger als noch 2009. Von Einzelfällen abgesehen, liegen diese Wahlkreise in NordrheinWestfalen (vor allem im Ruhrgebiet), in Nordhessen, Niedersachsen und in Hamburg. Die Linke konnte an ihren Erfolg von 2009 mit 16 Direktmandaten nicht anknüpfen; sie gewann lediglich die vier im ehemaligen Ostteil Berlins gelegenen Wahlkreise. Für die Grünen sicherte Hans-Christian Ströbele wie schon 2002, 2005 und 2009 das einzige Direktmandat in Berlin-Friedrichshain-Kreuzberg-Prenzlauer Berg-Ost. Er ist auch der Wahl- 
kreisgewinner mit der größten persönlichen Zugkraft. Sein Erstimmenergebnis liegt um 19,1 Prozentpunkte über dem Ergebnis seiner Partei. Das zweite von den Grünen anvisierte Direktmandat wurde jedoch verfehlt. Zwar erzielte Cem Özdemir im Wahlkreis 258 Stuttgart I ein Erststimmenplus von fast zehn Prozentpunkten, trotzdem unterlag er dem CDU-Kandidaten Stefan Kaufmann deutlich. Den zweitgrößten Erststimmenvorsprung verzeichnete Wolfgang Bosbach von der CDU. Im Wahlkreis 100 Rhein-Bergischer-Kreis lag er - ähnlich wie 2009 - 14,8 Prozentpunkte vor seiner Partei. Bundeskanzlerin Angela Merkel erhielt in ihrem Wahlkreis 15 Vorpommern-Rügen-Vorpommern-Greifswald I 11,3 Prozentpunkte mehr als der Zweitplatzierte. Auf Platz 3 der stärksten Direktkandidaten liegt Gregor Gysi von der Linken. In seinem Wahlkreis 84 Berlin-Treptow-Köpenick erzielte er einen Erststimmenvorsprung von 12,7 Prozentpunkten. Das größte Erststimmenplus bei den Sozialdemokraten verbuchte Ulrich Kelber im Wahlkreis 96 Bonn. Trotz der dortigen offiziellen Splitting-Absprachen zwischen FDP und CDU konnte er mit einem Plus von 12,2 Prozentpunkten vor seiner eigenen Partei den Wahlkreis, in dem auch Außenminister Guido Westerwelle angetreten war, knapp für die SPD gewinnen.

\subsection{Wahlverhalten nach Bevölkerungsgruppen}

2013 ist die Union dem Ideal einer Volkspartei, also breitem Rückhalt in den meisten Bevölkerungsgruppen, wieder näher gekommen. Zum einen konnte sie ihre Stimmenanteile in allen Bevölkerungsgruppen deutlich ausbauen. Zum anderen legte sie oft in jenen Gruppen besonders viel zu, in denen sie bisher vergleichsweise schwach war. Auch wenn ihr Wählerprofil nach wie vor die typischen Muster aufweist, ist es damit insgesamt etwas ausgeglichener als in den Jahren zuvor. Noch bei der Bundestagswahl 2009 schaffte die Union es bei Bürgern unter 60 Jahren gerade einmal auf knapp 30 Prozent, und bei einigen Landtagswahlen nahm die CDU bereits Züge einer Rentnerpartei an. Dank der überdurchschnittlichen Zuwächse vor allem in den mittleren Altersgruppen hat sich das Altersprofil aber etwas abgeflacht. Zwar erzielte sie nach wie vor ihre besten Ergebnisse bei Bürgern über 60 Jahren - jeder zweite (49 Prozent) in dieser Gruppe hat sich für die CDU/CSU entschieden; bei den älteren Frauen waren es sogar 53 Prozent. Aber auch in den mittleren Altersgruppen kam sie jetzt wieder an die 40-Prozent-Marke heran. Lediglich bei den 18- bis 24-Jährigen waren es - trotz Zuwächsen - nur 31 Prozent. Der 2009 durch leichte Gewinne bei den Frauen und deutliche Verluste unter den Männern entstandene GenderGap ist 2013 wegen gleichmäßiger Zuwächse bei beiden Geschlechtern erhalten geblieben. 44 Prozent der Frauen, aber nur 39 Prozent der Männer entschieden sich für die von der Kanzlerin angeführten Unionsparteien. Bei den Konfessionen hat sich das Gefälle reduziert. Zwar erreichte die Union unter den Katholiken die absolute Mehrheit (52 Prozent), unter den Protestanten konnte sie aber deutlich mehr zulegen (+10 Prozentpunkte). Wieder etwas klassischer ausgeprägt ist dagegen das Profil bei den Berufsgruppen. Durch besonders starke Zugewinne unter den Selbständigen (+15), die mit besonders starken Verlusten der FDP in dieser Gruppe korrespondieren, zählen die Selbständigen jetzt wieder zur Kerngruppe der CDU-Wähler. Unter den Arbeitern kann die Union den 2009 von der SPD eroberten ersten Platz behaupten und den Abstand zur SPD sogar etwas ausbauen (35 Prozent gegenüber 27). Lediglich unter den Arbeitslosen stagnierte der Wählerrückhalt der Union (+2 Prozentpunkte auf 24 Prozent). 
Bei der SPD hat sich das Wählerprofil gegenüber 2009 nur unwesentlich verändert. Wie die CDU/CSU erzielte sie ihre besten Resultate bei den Älteren, in den unteren Bildungsgruppen und unter Protestanten. Allerdings sind die Unterschiede nicht allzu groß. Überdurchschnittliche Zuwächse verzeichnete die SPD unter den Angestellten (+6 Prozentpunkte) und in den jüngeren Altersgruppen (+6 bei den 18- bis 24-Jährigen und +5 bei den 25- bis 34-Jährigen). Die dramatischen Einbrüche 2009 unter den Arbeitern (-13 Punkte) und unter den Angestellten (-15 Punkte), konnten damit aber bei Weitem nicht ausgeglichen werden. Durch die überdurchschnittlichen Gewinne der SPD bei den Jüngeren und ähnlich hohe Zuwächse der Union kommen die Volksparteien nach den drastischen Verlusten $2009^{46}$ auch bei den Jungwählern wieder zusammengenommen auf deutlich über 50 Prozent.

Die Liberalen verloren in allen Bevölkerungsgruppen stark, am meisten unter den Selbständigen (-16 Prozentpunkte). Gleichwohl sind diese nach wie vor die Kerngruppe der FDP-Wähler. Hier schaffte es die Partei immer noch auf zehn Prozent. In allen anderen Bevölkerungsgruppen rangieren die Liberalen nahe an der Fünf-Prozent-Hürde, bei Frauen, Arbeitern, Beamten sowie den unteren und mittleren Bildungsgruppen sogar teils deutlich darunter.

Die Linke schneidet nach wie vor unter Arbeitslosen und Arbeitern am stärksten ab. Fast ein Viertel (23 Prozent) der Arbeitslosen hat sich für die Linke entschieden, fast genauso viele wie für Union (24 Prozent) und SPD (26 Prozent). Dennoch musste die Linke in allen Bevölkerungsgruppen Verluste hinnehmen, etwas größere in den mittleren Altersgruppen und bei den Arbeitern, etwas kleinere bei den Bürgern über 60 Jahren und in den oberen Bildungsgruppen. Auch bei den Arbeitslosen fallen die Verluste nur unterdurchschnittlich aus, allerdings hat sich deren Zahl insgesamt seit 2005 fast halbiert. Neben den starken Niveauunterschieden zwischen West und Ost sind auch die unterschiedlichen Altersprofile bemerkenswert. Während in Ostdeutschland die Linke ihre besten Ergebnisse unter den Älteren (ab 45 Jahren) erzielte, schnitt sie im Westen bei den Jüngeren merklich besser ab als bei den Bürgern über 60 Jahren.

Das Sozialprofil der Grünen-Wähler hat sich wenig verändert. Wie bei den Wahlen zuvor erzielten sie 2013 überdurchschnittliche zweistellige Ergebnisse bei Bürgern unter 60 Jahren, bei den Frauen, in den oberen Bildungsgruppen und unter Angestellten, Beamten und Selbständigen. Verluste mussten sie - wie FDP und Linke - aber in allen Gruppen verkraften. Auffällig ist, dass diese in ihren Kerngruppen etwas stärker ausfielen, so dass sich die Unterschiede etwas verringert haben.

Die AfD schnitt bei Jüngeren und bei Männern deutlich besser ab als unter den Älteren und den Frauen und zeigt damit das typische Muster von Protestparteien. Bei den Männern schaffte die AfD in allen Altersgruppen unter 60 Jahren deutlich den Sprung über die Fünf-Prozent-Hürde. Bei den Frauen liegt sie entweder an oder unter der Fünf-ProzentHürde. Bei den Bürgern über 60 Jahren genießt die AfD sowohl unter Frauen (zwei Prozent) als auch unter Männern (vier Prozent) deutlich weniger Rückhalt. Auf wenig Resonanz stieß die Partei auch unter Arbeitslosen (drei Prozent). Zwischen den Berufsgruppen zeigen sich dagegen kaum Unterschiede. 


\subsection{Parteikompetenzen und Wahlmotive}

Die Union profitierte massiv von der guten wirtschaftlichen Lage und der positiven Bewertung der Bundesregierung. Ihr ist es insbesondere gelungen, sämtliche Unzufriedenheit mit der Regierung beim liberalen Koalitionspartner abzuladen. Inhaltlich war sie - in den Augen der Wähler - schon lange nicht mehr so gut aufgestellt wie vor dieser Wahl. Am deutlichsten wurde dies bei der Wirtschaftspolitik. Sechs von zehn Wahlberechtigten (58 Prozent) hielten am ehesten die CDU/CSU für fähig, die Wirtschaft in Deutschland voranzubringen - ein Zuwachs gegenüber der letzten Bundestagswahl von elf Prozentpunkten. Noch stärker (+14 Prozentpunkte) konnten sich die Unionsparteien bei der Frage nach Erhalt und Schaffung neuer Arbeitsplätze verbessern. Jeder Zweite (51 Prozent) traute ihnen in diesem Bereich am meisten zu (vgl. Tabelle 1). Ähnlich klar dominierten die Christdemokraten das Feld bei der Haushalts- und Finanzpolitik (48 Prozent), der Außenpolitik (50 Prozent) und der Eurokrisenpolitik (46 Prozent). Damit konnten sie in ihren Kernkompetenzfeldern den 2009 gegenüber der FDP verlorenen Boden wieder gutmachen und darüber hinaus sogar mehr Zustimmung gewinnen als bei allen anderen Bundestagswahlen nach der Helmut-Kohl-Ära. Die Union konnte aber nicht nur ihre Stärken ausbauen, sondern auch ihre Schwächen abmildern. Sowohl bei sozialen Gerechtigkeitsthemen als auch - dank Atomausstieg und Energiewende - bei Umweltfragen verzeichneten CDU und CSU mehr Zuspruch der Bürger als noch 2009 („für soziale Gerechtigkeit sorgen“ und „für angemessene Löhne sorgen“ jeweils +5, „gute Umweltpolitik“+7 Punkte). Der Abstand zu den Sozialdemokraten bei sozialen Themen und zu den Grünen bei ökologischen Fragen hat sich damit deutlich verringert.

$\mathrm{Zu}$ diesem deutlich breiteren thematischen Kompetenzprofil kam die gute Bewertung des politischen Personals der Union, allen voran die alle überstrahlende Bundeskanzlerin, mit der parteiübergreifend fast drei Viertel der Bundesbürger (71 Prozent) zufrieden waren. Jeweils eine breite Mehrheit hielt das Handeln Merkels in der Eurokrise für richtig und entschlossen (59 Prozent), empfand ihre Regierungsführung als überparteilich (60 Prozent) und schätzte ihr Auftreten auf internationaler Bühne (84 Prozent). Entsprechend hätte eine deutliche Mehrheit (58 Prozent) der Bürger für Angela Merkel als Bundeskanzlerin gestimmt, wenn sie darüber direkt hätten entscheiden können. Die Zugkraft der Regierungschefin hat bei dieser Wahl noch einmal deutlich zugenommen. 38 Prozent der Unionswähler gaben in der Infratest-dimap-Wahltagsbefragung an, sie hätten ihre Entscheidung in erster Linie wegen der Spitzenkandidatin getroffen - bei der Bundestagswahl 2009 waren es 32 Prozent, bei ihrer ersten Wahl nur 16 Prozent. Auch die Zuwächse gingen vornehmlich auf das Konto der Bundeskanzlerin: Unter den neu gewonnenen Unionswählern nannten vier von zehn die Spitzenkandidatin als wahlentscheidend. Aber auch in der zweiten Reihe überzeugte die CDU/CSU mit ihrem Personalangebot. Finanzminister Wolfgang Schäuble konnte als Garant für solides Wirtschaften und eine besonnene Euro-Finanzpolitik punkten und breite Zustimmung zu seiner Politik verzeichnen (62 Prozent). Selbst CSU-Chef Horst Seehofer, der auf Bundesebene durchaus auch den Konflikt mit der Kanzlerin suchte, wurde nicht nur in Bayern, sondern auch bundesweit mehrheitlich positiv bewertet (53 Prozent).

Von diesem ausgesprochen günstigen Erscheinungsbild konnte die Regierungskoalition als Ganzes nur bedingt profitieren. Die Neigung der Unionsanhänger zu Schwarz-Gelb war gegenüber 2009 deutlich abgekühlt. Zwar hätte immer noch eine klare Mehrheit (66 Prozent) eine Fortsetzung der alten Koalition begrüßt, allerdings wünschte sich immerhin ein 
Drittel der Unionsanhänger (32 Prozent) einen Wechsel des Koalitionspartners. Entsprechend stößt die mittlerweile gebildete Große Koalition unter ihnen auf breite Zustimmung. Sofern es für eine Fortsetzung der alten Regierung nicht reichen sollte, wünschten sich (am Wahltag) 69 Prozent der Unionswähler eine Große Koalition, nur 19 Prozent hätten ein Zusammengehen mit den Grünen bevorzugt.

Die SPD schien sich im Laufe der Wahlperiode von den innerparteilichen Querelen und dem personellen und koalitionstaktischen Durcheinander während der vorangegangenen Großen Koalition erholt zu haben und konnte wieder Vertrauen in ihre Problemlösungskompetenz zurückgewinnen. Auch traf sie mit den im Wahlkampf propagierten Themen die Kritik weiter Teile der Bevölkerung an der Politik der Bundesregierung. Nach dem langen Wahlkampf standen die Sozialdemokraten aber wieder ähnlich schwach da wie vor der letzten Bundestagswahl. Ihr Markenkern „soziale Gerechtigkeit“ ist weiterhin beschädigt. Nicht einmal jeder zweite Bürger (43 Prozent) traut ihnen hier am meisten zu. Damit liegt die SPD in diesem Bereich - genauso wie beim Kampf für angemessene Löhne - zwar klar vor allen anderen Parteien, konnte allerdings damit bei weitem nicht an die Werte der ersten beiden Wahlen mit dem Spitzenkandidaten Gerhard Schröder anknüpfen (54 und 52 Prozent), sondern verharrte auf dem Niveau seit Umsetzung der Agenda 2010 (vgl. Tabelle 1). Auch wenn zwei Drittel der Bürger der SPD und ihrer Agendapolitik Verdienste um die gute wirtschaftliche Lage in Deutschland bescheinigen (65 Prozent), sehen ähnlich viele (62 Prozent) in Hartz IV und der Rente mit 67 eine Abkehr von sozialdemokratischen Prinzipien. Der Partei ist es nicht gelungen, diesen Widerspruch aufzulösen. Bei der Wirtschaftskompetenz lässt sich ebenfalls - trotz des Spitzenkandidaten Peer Steinbrück - kein Wiedererstarken erkennen. Augenhöhe mit der Union ist in diesem Politikfeld traditionell für die SPD nicht zu erreichen; allerdings lagen die Werte unter Gerhard Schröder 1998 und 2002 um elf beziehungsweise fünfzehn Prozentpunkte höher, und der Abstand zur CDU/CSU war mit 36 Prozentpunkten in den letzten 15 Jahren noch nie so groß wie bei dieser Wahl. Zu den besonders umkämpften Politikfeldern zwischen Union und SPD zählt traditionell die Arbeitsmarktpolitik. Konnte die von Schröder geführte SPD die Union 1998 in diesem Politikfeld noch klar übertrumpfen (43 zu 28 Prozent) und 2002 immerhin noch Augenhöhe herstellen (38 zu 41 Prozent), so musste sie danach die Union an sich vorbei ziehen lassen. 2013 hat sich der Rückstand noch einmal deutlich auf 22 Prozentpunkte erhöht (29 zu 51 Prozent). In den zentralen Politikbereichen Wirtschaft, Arbeit und Soziales ist damit für die Sozialdemokraten gegenüber 2009 lediglich Stagnation zu diagnostizieren (vgl. Tabelle 1). Deutliche Zugewinne (+7 Punkte) können sie nur bei der Familienpolitik und der Kinderbetreuung verzeichnen, also genau dort, wo die CDU von ihrem früheren Modernisierungskurs abgewichen ist. Die in diesem Feld deutlich konservativere Positionierung der Union, symbolisiert durch den Ministerwechsel von Ursula von der Leyen zu Kristina Köhler (später Schröder) und das von der CSU durchgesetzte Betreuungsgeld ließen der SPD Raum für eine bessere Positionierung als 2009.

Auch auf personeller Ebene konnte die SPD nicht punkten. Steinbrück hatte lange Mühe, sich beim Einsatz für soziale Gerechtigkeit von der Kanzlerin deutlich abzusetzen. Erst gegen Ende des Wahlkampfes konnte er eine klare Mehrheit der Bürger überzeugen, in diesem Bereich der bessere Kandidat zu sein (51 gegenüber 33 Prozent für Merkel). Bis zuletzt litt sein Wahlkampf jedoch unter mangelnder Glaubwürdigkeit auf diesem Feld. Immerhin vier von zehn Bürgern (42 Prozent) waren der Meinung, dass Steinbrück eigentlich nicht richtig zur SPD passe. Zugleich gelang es der SPD und ihrem Spitzenkandidaten 


\begin{tabular}{|c|c|c|c|c|c|}
\hline $\begin{array}{c}\text { Tabelle 1: Parteikompetenzen von Union und SP } \\
\text { Gerechtigkeit } 1998 \text { bis } 2013\end{array}$ & auf $d$ & ielder & rtsch & Irbei & soziale \\
\hline Welcher Partei trauen Sie am ehesten zu ... & 1998 & 2002 & 2005 & 2009 & 2013 \\
\hline ... die Wirtschaft in Deutschland voranzubringen? & & & & & \\
\hline Union & 47 & 46 & 51 & 47 & 58 \\
\hline SPD & 33 & 37 & 27 & 21 & 22 \\
\hline ... Arbeitsplätze zu sichern und neue zu schaffen? & & & & & \\
\hline Union & 28 & 41 & 45 & 37 & 51 \\
\hline SPD & 43 & 38 & 25 & 31 & 29 \\
\hline ... für soziale Gerechtigkeit zu sorgen? & & & & & \\
\hline Union & 20 & 25 & 28 & 19 & 24 \\
\hline SPD & 54 & 52 & 42 & 44 & 43 \\
\hline
\end{tabular}

nicht, dessen Kompetenz in Wirtschafts- und Finanzfragen erfolgreich ins Spiel zu bringen. Trotz positiver Bewertung seiner früheren Arbeit als Finanzminister glaubte nicht einmal jeder Zweite (45 Prozent), dass er in der Lage sei, das Land gut zu führen. Beim wirtschaftspolitischen Sachverstand musste er der Bundeskanzlerin im Direktvergleich den Vortritt lassen (38 zu 43 Prozent), und beim Zutrauen in die Bewältigung der Euro- und Schuldenkrise wurde er sogar deklassiert (25 zu 52 Prozent). Entsprechend konnte Steinbrück an den Wahlurnen nur wenig Zugkraft entwickeln. Lediglich 21 Prozent der SPD-Wähler nannten den Spitzenkandidaten als entscheidenden Grund für ihre Stimmabgabe, ein fast identischer Anteil wie 2009 für Frank-Walter Steinmeier - bei Schröder waren es noch um die 40 Prozent gewesen.

Das klare Ergebnis des SPD-Mitgliederentscheides für die Große Koalition im Dezember war am Wahltag noch nicht abzusehen. Zwar wünschte sich eine relative Mehrheit von 43 Prozent der SPD-Wähler eine Große Koalition, sollte es weder für Schwarz-Gelb noch für Rot-Grün zum Regieren reichen. Immerhin 33 Prozent der SPD-Wähler präferierten zum damaligen Zeitpunkt jedoch ein Zusammengehen mit den Grünen und der Linken.

Die Liberalen traten sowohl inhaltlich als auch personell völlig ausgezehrt vor die Wähler. Von dem vor der Bundestagswahl 2009 breiten Kompetenzprofil der FDP war unmittelbar vor der Wahl 2013 kaum noch etwas übrig. Nur noch sechs Prozent bescheinigten ihr den größten Sachverstand in der Steuerpolitik (-13 Prozentpunkte) und gerade einmal drei Prozent attestierten ihr - trotz Ressortverantwortung - die meiste Wirtschaftskompetenz (-11 Punkte). Auch bei der Außenpolitik (sechs Prozent) und der Gesundheitspolitik (vier Prozent) gelang es den Liberalen nicht, ihre ministerielle Zuständigkeit zu nutzen und in den Augen der Bürger inhaltliche Kompetenz unter Beweis zu stellen. Mit der Arbeit der FDP in der Bundesregierung waren gerade einmal zwölf Prozent zufrieden. Auch das Personal überzeugte die Bürger nicht: Parteichef Philipp Rösler und Spitzenkandidat Rainer Brüderle zählten regelmäßig zu den unbeliebtesten deutschen Spitzenpolitikern. Außenminister Guido Westerwelle konnte zwar durchaus im Lauf der Wahlperiode an Ansehen gewinnen, so dass sich unmittelbar vor der Wahl Zustimmung und Ablehnung seiner politischen Arbeit die Waage hielten. An die hohe Reputation seiner Amtsvorgänger Steinmeier und Fischer konnte Westerwelle jedoch nie anknüpfen. 
Mangels inhaltlicher und personeller Überzeugungskraft setzte die FDP am Ende nahezu ausschließlich auf eine Zweitstimmenkampagne. Auch wenn es für den Sprung über die Fünf-Prozent-Hürde letztlich nicht reichte, blieb diese Kampagne nicht ohne Resonanz. Fast jeder zweite FDP-Wähler (46 Prozent) hat sich nur aus Koalitionstaktik für die Liberalen entschieden und bevorzugte eigentlich eine andere Partei. Zum Vergleich: Bei den anderen im Bundestag vertretenen Parteien nannten nur zwischen neun und 19 Prozent Koalitionstaktik als Hauptargument.

Die Vernachlässigung ökologischer Themen im Wahlkampf schlug sich im Kompetenzprofil der Grünen deutlich nieder. Zwar waren sie nach wie vor die Partei, der die meisten Bürger die beste Umweltpolitik zutrauen (56 Prozent), allerdings überzeugten sie damit erheblich weniger als noch 2009 (-8 Prozentpunkte). Beim Einsatz für sichere und bezahlbare Energie setzte noch ein gutes Viertel auf die Grünen (27 Prozent); im Fukushima-Jahr 2011 waren es zeitweise über 35 Prozent. Während sich die Vernachlässigung urgrüner Themen negativ auswirkte, führte die Schwerpunktsetzung auf soziale Fragen und Verteilungsgerechtigkeit - bei denen die Bürger eher auf SPD und Linke setzen - zu keinen Kompetenzgewinnen. Nur fünf Prozent vertrauten in diesem Bereich den Grünen, genauso viele wie 2009. Auch beim Einsatz für ein gerechtes Steuer- und Abgabensystem präferierten nur sechs Prozent die Grünen. Ihre Steuerpläne wirkten eher abschreckend, zumindest sahen dies zwei Drittel der Bundesbürger (68 Prozent) und fast die Hälfte (44 Prozent) der Grünen-Anhänger so.

Den grünen Negativtrend im Laufe des Wahlkampfes konnte auch der Spitzenkandidat Jürgen Trittin nicht bremsen. Als potentieller Finanzminister einer rot-grünen Koalition konzentrierte er sich weit stärker auf soziale und steuerpolitische Themen als auf ökologische, mit der Folge, dass seine Zustimmungswerte kontinuierlich sanken. Nachdem unmittelbar vor der Wahl der Göttinger Politologe Franz Walter eine öffentliche Diskussion über Trittins Rolle in der Pädophilie-Debatte ${ }^{47}$ angestoßen hatte, erhielt er zuletzt nur 36 Prozent. Die zweite Spitzenkandidatin, Katrin Göring-Eckardt, konnte diesen Sympathieverlust nicht kompensieren.

Der spätere Abbruch der Sondierungsgespräche zwischen Grünen und Union dürfte nicht nur den inhaltlichen und kulturellen Differenzen auf Ebene der Parteieliten geschuldet sein, sondern auch der verbreiteten Skepsis grüner Wähler gegenüber einer Koalition mit der Union. Fast die Hälfte optierte am Wahltag, sofern es nicht für Rot-Grün oder Schwarz-Gelb reichen sollte, für ein Bündnis mit SPD und Linken (44 Prozent). Nur jeder Fünfte (19 Prozent) plädierte für Schwarz-Grün. Fast genauso viele (17 Prozent) wünschten sich für diesen Fall mit der Großen Koalition eine Regierung ohne die eigene Partei.

Zu den Aufsteigern dieses Wahlkampfes zählt zweifelsohne der Spitzenkandidat der Linken, Gregor Gysi. Während er im Zufriedenheitsranking der Spitzenpolitiker den Großteil der Wahlperiode im unteren Drittel verbrachte, konnte er im Laufe der Kampagne deutlich mehr Bürger überzeugen. Unmittelbar vor der Wahl fällte fast jeder zweite (47 Prozent) ein positives Urteil über Gysis politische Arbeit. Damit war er nach der Bundeskanzlerin der zweitpopulärste Spitzenkandidat. Inhaltlich-programmatisch veränderte sich das Profil der Linken gegenüber 2009 kaum. Trotz leichter Rückgänge lag ihr Kompetenzschwerpunkt in

47 Das Göttinger Institut für Demokratieforschung war vom Bundesvorstand der Grünen beauftragt worden, den Stellenwert pädophiler Forderungen in den Milieus der Neuen Sozialen Bewegungen sowie der Grünen insbesondere Anfang der 1980er Jahre zu untersuchen. 
den Augen der Bürger nach wie vor beim Einsatz für soziale Gerechtigkeit (zwölf Prozent) und für angemessene Löhne (elf Prozent). Die Konzentration auf ihre Kernkompetenzen hat der Linken deutlich geholfen. War ihr Einzug in den Bundestag am Beginn des Wahljahres zwar wahrscheinlich, aber keineswegs gesichert, so reichte es am Ende sogar knapp auf den dritten Platz vor den Grünen. Trotz der klaren inhaltlichen Kompetenzschwerpunkte schwingt bei vielen Bürgern (insbesondere im Westen) immer noch ein starkes Protestmotiv bei der Wahl der Linken mit. Fast jeder zweite ihrer Wähler (43 Prozent) entschied sich nicht aus Überzeugung für sie, sondern aus Enttäuschung über andere Parteien.

Den stärksten Endspurt im Wahlkampf legte die neu gegründete AfD hin. Den ganzen Sommer über war sie weit von der Fünf-Prozent-Hürde entfernt, obwohl sie eine für eine bisher weder im Bundestag noch in einem einzigen Landtag vertretene Partei ungewöhnlich starke Medienpräsenz vorweisen konnte. Unmittelbar vor der Wahl trug diese Präsenz dann doch Früchte: Unter den Wählern, die sich erst in den letzten Tagen oder am Wahltag selbst entschieden hatten, erzielte die AfD mit sieben Prozent ein weit überdurchschnittliches Ergebnis. ${ }^{48}$ Die Partei konnte im Wahlkampf mit ihrer euro-kritischen Agenda durchaus an eine weit verbreitete Verunsicherung anknüpfen. Fast alle Bürger (89 Prozent) gingen von deutlich höheren Kosten der Staatsschulden- und Finanzmarktkrise im Euro-Raum für die deutschen Steuerzahler aus, als den bis dahin öffentlich diskutierten; viele - vor allem Wähler der AfD - fürchteten um ihre Ersparnisse. Fast jeder zweite (44 Prozent) bescheinigte der AfD beim Euro immerhin eine klare Benennung der Probleme. Allerdings war das Vertrauen der Bürger, dass sie diese Probleme auch erfolgversprechend angehen könne, äußerst begrenzt. Nur drei Prozent hielten die AfD am ehesten für in der Lage, die Krise in den Griff zu bekommen. In allen andern Politikfeldern konnte sie überhaupt nicht punkten. Dazu passend wählte auch nur ein gutes Drittel der AfD-Wähler (37 Prozent) ihre Partei aus Überzeugung. Für die meisten (57 Prozent) war Enttäuschung über andere Parteien das Hauptmotiv für ihre Wahlentscheidung.

\section{Der lange Weg zur dritten Großen Koalition}

Die Wahl vom 22. September mündete in eine schwierige Regierungsbildung, die länger dauerte als jemals zuvor. ${ }^{49}$ Erst drei Monate nach der Wahl wurde das neue Kabinett vereidigt. Das Problem lag dabei nicht am Wahlergebnis selbst, dafür waren der Wahlsieg der Union und das Votum für Merkels dritte Amtszeit zu eindeutig. Zwar sah sich die Union einer knappen Mehrheit linker Parteien gegenüber, aber das „hinkende“ 50 linke Lager erwies sich nach wie vor als nicht einmal ansatzweise koalitionsfähig; es reichte nicht einmal für Sondierungsgespräche. Bereits am Wahlabend war klar, dass Merkel weiter regieren würde, offen blieb nur, mit wem. Ihr Wunschpartner FDP stand nicht mehr zur Verfügung, sie

48 Ein überdurchschnittliches Ergebnis unter Spätentscheidern ist für Protest- und Kleinparteien nicht ungewöhnlich, vgl. Stefan Merz, Das Kreuz mit dem Kreuz: Unentschlossene Wähler in Deutschland, Baden-Baden 2010, S. 159 - 163.

49 Ausführlich zur Regierungsbildung siehe den Beitrag von Roland Sturm in diesem Heft.

50 Joachim Raschke / RalfTils, Der steinige Weg zur Macht, Heinrich Böll Stiftung, 26. April 2013, http://www.boell.de/de/node/277255 (Abruf am 20. Januar 2014). 
hatte nur die Wahl zwischen der SPD und den Grünen, die die angestrebte gemeinsame Mehrheit deutlich verfehlt hatten. In beiden Parteien gab es allerdings deutliche Vorbehalte gegen ein Bündnis mit der Union. Bei der SPD gründete sich die Ablehnung vor allem in der Befürchtung, wie nach der letzten Koalition mit der Merkel'schen Union vom Wähler abgestraft zu werden - wobei meist unterschlagen wurde, dass das deprimierende Wahlergebnis von 2009 weniger der Regierungsbeteiligung der SPD galt als vielmehr ihrem innerparteilichen Zwist und anhaltenden Personalquerelen. Bei den Grünen überwogen zumindest in der öffentlichen Diskussion inhaltliche Vorbehalte. $\mathrm{Zu}$ groß schienen die Gegensätze vor allem im Bereich der Steuerpolitik, wo sich die Grünen in der Wahl stärker noch als die SPD für höhere Abgaben eingesetzt hatten, und in der Gesellschaftspolitik, wo die Gegensätze vor allem zur CSU (Betreuungsgeld, Gleichstellungspolitik) grundsätzlicher Natur waren. Ausschlaggebend für den Rückzug der Grünen aus den Sondierungsgesprächen war aber auch der umfassende Personalumbau, den sich die Partei nach der überraschenden Wahlniederlage selbst verordnete. Dabei auch noch eine schwierige und auf Bundesebene unerprobte Koalition mit der Union einzugehen, stellte offenbar ein zu großes Risiko dar. ${ }^{51}$

Nach dem Rückzug der Grünen lief alles auf eine Wiederauflage der Großen Koalition hinaus - für die Union sicherlich die strategisch weniger reizvolle, dafür aber programmatisch naheliegende Option. Bei der SPD mussten allerdings noch einige Hürden genommen werden. Die Zustimmung der Parteigremien einzuholen war davon die leichtere Aufgabe. Gabriel erschien es aber ratsam, die neue Koalition auch auf dem Fundament eines positiven Mitgliedervotums aufzubauen. Letztlich erwies sich das als geschickter Schachzug Gabriels gleich in dreifacher Hinsicht. Erstens trug es erheblich zur innerparteilichen Befriedung der SPD nach dem enttäuschenden Wahlergebnis bei und hatte einen Anstrich von Neustart. Zweitens verhalf die Einbeziehung der Mitglieder als Letztinstanz den Sozialdemokraten zu einer Verhandlungsposition auf Augenhöhe gegenüber der nach Stimmen deutlich mächtigeren Union. Drittens festigte die deutliche Zustimmung die Führungsposition des Vorsitzenden, zumal diesmal - anders als 2005 - auch die erweiterte Führungsmannschaft und die Mitglieder in die Entscheidung und damit auch in die Verantwortung eingebunden wurden.

Die Rollenverteilung in den Verhandlungen war etwas eigentümlich. SPD und CSU brachten offensiv ihre im Wahlkampf vertretenen Positionen und Forderungen vor. Für die SPD waren vor allem Mindestlohn und abschlagsfreie Rente nach 45 Beitragsjahren nicht verhandelbar, bei der CSU das Betreuungsgeld sowie die PKW-Maut für Ausländer. Die CDU trat dagegen eher defensiv auf (keine Steuererhöhung, keine Neuverschuldung); sie hatte ihr zentrales Wahlkampfziel - Merkels dritte Amtszeit - schon erreicht. Dies erleichterte notwendige Kompromisse, zumal manche Forderung eher vage und damit verhandlungsoffen formuliert wurde (,verbindliche Regelungen für mehr Frauen in Führungspositionen"). Gleichwohl gestalteten sich die Verhandlungen in den zwölf thematisch definierten Arbeitsgruppen und vier Untergruppen mit insgesamt knapp 100 Teilnehmern teilweise recht schwierig.

51 Wolfgang Schäuble kommentierte das süffisant in der FAS vom 22. Dezember 2013, „Ich sah den Angstschweiß der Grünen", http://www.faz.net/aktuell/politik/inland/wolfgang-schaeuble-imgespraech-ich-sah-den-angstschweiss-der-gruenen-12721918.html (Abruf am 13. Januar 2014). 
Nach wochenlangen Verhandlungen stand am Ende ein insgesamt 185 Seiten umfassender Koalitionsvertrag ${ }^{52}$ mit dem eher unverbindlichen Titel „Deutschlands Zukunft gestalten“. Der Vertrag enthält eine Reihe sehr konkreter Vereinbarungen (Mindestlohn von 8,50€, Mütterrente, Renteneintritt mit 63, doppelte Staatsbürgerschaft), an konkrete Bedingungen geknüpfte Maßnahmen (Ausländermaut) sowie Absichtserklärungen (Gleichstellung, Datenschutz). Manches blieb unklar, wie etwa Ausnahmeregelungen beim Mindestlohn.

Unklar war bis zum Schluss, ob die SPD-Mitglieder dem Koalitionsvertrag zustimmen würden - einem Vertrag, zu dem die große Mehrheit der Bundesbürger Zustimmung signalisierte. ${ }^{53}$ In zahlreichen Regionalkonferenzen, auf denen die Parteispitze um Zustimmung warb, wurden allerdings Vorbehalte vieler SPD-Mitglieder gegen eine Koalition mit der Union deutlich, weshalb der Ausgang der Abstimmung bis zum Schluss als ziemlich offen galt. Die Erleichterung war - nicht nur auf SPD-Seite - groß, als die Stimmauszählung eine deutliche Mehrheit auch der Mitglieder für die Annahme des Vertrages erbrachte. Bei einer ebenfalls unerwartet hohen Beteiligungsrate von 78 Prozent gaben 75,96 Prozent der SPD-Mitglieder ein positives Votum ab. Mit diesem neuen partizipativen Element brachte die SPD nicht nur neuen Schwung in ihre innerparteilichen Strukturen und Abläufe, sie löste auch eine öffentliche Debatte über Sinnhaftigkeit, Legitimität und Legalität des Verfahrens aus. ${ }^{54}$ Mit dem überraschend positiven Ausgang des Entscheids war die öffentliche Debatte darüber aber weitgehend beendet. Offen bleibt, ob dieses Verfahren auch bei künftigen Koalitionsverhandlungen zur Anwendung kommen wird.

Da bereits vor dem Mitgliederentscheid der Koalitionsvertrag vom Bundesausschuss der CDU und vom Parteivorstand der CSU gebilligt worden war, stand der Vereidigung der Kanzlerin am 17. Dezember und der Mitglieder ihres Kabinetts am Tag darauf nichts mehr im Wege. Die Besetzung des Kabinetts blieb bis zum Vorliegen des SPD-Mitgliedervotums geheim. Es waren viele erwartete Kandidaten darunter, die Besetzungsliste enthielt aber auch Überraschungen, allen voran Ursula von der Leyen als neue und erste weibliche Verteidigungsministerin. Die CDU erhielt weitere vier Ministerien, die mit Thomas de Maizière (Inneres), Wolfgang Schäuble (Finanzen), Hermann Gröhe (Gesundheit) und Johanna Wanka (Bildung und Forschung) besetzt wurden, sowie den Posten des Kanzleramtsministers, den Peter Altmaier übernahm. Sechs Ministerien gingen an die SPD: Sigmar Gabriel, erhielt das um die Energiepolitik erweiterte Wirtschaftsministerium und amtiert als stellvertretender Regierungschef; hinzu kommen Frank-Walter Steinmeier (Außen), Andrea Nables (Arbeit und Soziales), Manuela Schwesig (Familie), Heiko Maas (Justiz) und Barbara Hendricks (Umwelt). Die CSU stellt wie im 17. Bundestag drei Minister, musste allerdings das wich-

52 Zum Vergleich: Der Koalitionsvertrag der letzten großen Koalition umfasste 160 Seiten, der der schwarz-gelben Koalition nur 134 Seiten.

53 In einer Umfrage von Infratest dimap für „Bericht aus Berlin“ Anfang Dezember 2013 sprachen sich 66 Prozent der Gesamtbevölkerung und sogar 75 Prozent der SPD-Anhänger für die Annahme des Koalitionsvertrages aus.

54 Vgl. exemplarisch Dietmar Neuerer, Staatsrechtler stellt SPD-Befragung zum Koalitionsvertrag infrage, in: Handelsblatt vom 28. November 2013, http://www.handelsblatt.com/politik/ deutschland/bundestagswahl-2013/verfassungsrechtlich-nicht-legitim-staatsrechtler-stellt-spdbefragung-zum-koalitionsvertrag-infrage/9139738.html; Christoph Seils, Das Grundgesetz und Frau Slomkas peinliche Fragen, in: Cicero vom 29. November 2013, http://www.cicero.de/berliner-republik/spd-mitgliederentscheid-die-quatsch-these-von-frau-slomka/56530 (jeweils Abruf am 19. Februar 2014). 
tige Innenministerium an die Schwesterpartei abgeben. Hans-Peter Friedrich wechselte in das Landwirtschaftsministerium, Gerd Müller übernahm das Amt des Entwicklungsministers und der neue starke Mann in der Bundes-CSU, Alexander Dobrindt, das Verkehrsministerium.

Von den beiden Oppositionsparteien im Bundestag hatte die Linke weitaus einfachere Startbedingungen. Sie konnte sich sofort in die neue Rolle als stärkste Oppositionspartei eingewöhnen, da sie mit weitgehend unverändertem Führungspersonal weitermachen konnte. Gysi ist und bleibt die Gallionsfigur der Linken, im neuen Bundestag sogar ausgestattet mit der Funktion des Oppositionsführers - bislang ohne zweite Person, wie im Fraktionsstatut eigentlich vorgesehen. Die einzige ernsthafte Kandidatin, Sarah Wagenknecht, akzeptiert Gysi nicht. Nach „seinem“ Wahlsieg ist Gysis Position so stark, die Nachfolgefrage damit aber auch so brisant wie nie zuvor. Damit verbunden ist die Frage der Koalitionsfähigkeit. Nachdem Gabriel per Parteitagsbeschluss die prinzipielle Absage an eine Koalition mit der Linken im Bund kassiert hat, liegt der Ball nun bei der Linkspartei.

Die Grünen stecken mitten im personellen Umbruch, denn das Ende der 68er Generation ist mit dem Rückzug Jürgen Trittins vom Fraktionsvorsitz endgültig vollzogen. Mit Claudia Roth und Renate Künast traten zwei weitere Führungsfiguren ins zweite Glied zurück, von der alten Viererspitze blieb nur Cem Özdemir als Parteivorsitzender. Als neue Vorsitzende wählte der Parteitag im Oktober 2013 die ehemalige saarländische Umweltministerin Simone Peters. Die von 68 auf 63 reduzierten grünen Bundestagsabgeordneten wählten in einer Kampfabstimmung die Spitzenkandidatin des Wahlkampfs, Katrin Göring-Eckardt, sowie den dem linken Lager zugerechneten Anton Hofreiter an die Spitze ihrer Fraktion. Als erste schwere Bewährungsprobe musste die neue Parteispitze die Sondierungsgespräche mit der Union leiten, unterstützt allerdings noch von den alten Führungskräften. Man fand zwar keine „belastbare Grundlage für vier Regierungsjahre“ "55, aber die Gesprächsatmosphäre wurde von beiden Seiten als „,außerordentlich sachlich, sehr neugierig, klug und konstruktiv" 56 bewertet. Konkrete Ergebnisse brachte die neue Bereitschaft zum Dialog in Hessen, wo eine schwarz-grüne Regierung gebildet wurde - die zweite nach Hamburg, die erste in einem Flächenland. Sollte diese Regierung erfolgreicher sein als das Vorgängermodell in Hamburg, ergeben sich daraus für beide Seiten neue strategische Koalitionsoptionen.

Das Scheitern der FDP hatte einen noch umfassenderen personellen Wechsel zur Folge. Das gesamte politische Führungspersonal zog sich aus der ersten Reihe der Partei zurück, der Vorsitzende Rösler sogar aus der Politik insgesamt. Auf ihrem Bundesparteitag im Dezember 2013 wurde eine völlig neue Führungsmannschaft gewählt mit Christian Lindner an der Spitze. Sein mäßiges Wahlergebnis von 79 Prozent lässt Zweifel daran erahnen, ob mit ihm als Parteivorsitzenden die Rückkehr in den nächsten Bundestag gelingen wird. Schwierig wird es allemal, denn die FDP kann in keinem Politikfeld den Alleinanspruch für liberale Politik mehr beanspruchen. Selbst ihre außerparlamentarischen Wettbewerber AfD und Piratenpartei stellen - auf unterschiedlichen Gebieten - ernstzunehmende Konkurrenten dar. Wie die FDP unter diesen erschwerten Bedingungen bestehen wird, wird

55 „Schöne Gespräche“ - aber die Grünen wollen nicht, in: Süddeutsche.de vom 16. Oktober 2013, http://www.sueddeutsche.de/politik/gescheiterte-schwarz-gruene-sondierung-schoene-gespraeche-aber-die-gruenen-wollen-nicht-1.1795837 (Abruf am 13. Januar 2014).

56 Ebenda. 
sich schon bei der Wahl zum Europäischen Parlament und bei den drei Landtagswahlen im Herbst 2014 erweisen. In Sachsen geht es immerhin um die Verteidigung der letzten verbliebenen Regierungsbeteiligung der Liberalen.

Die Ausgangslage für die AfD ist im Vergleich dazu gar nicht so schlecht: Sie hat in Bernd Lucke eine weitgehend unumstrittene Führungsfigur und ein klares, wenn auch thematisch sehr eingeengtes Profil. Bei der anstehenden Europawahl könnte dies ein Vorteil sein, zumal ihre Chancen auf den ersten Einzug in ein Parlament erheblich gestiegen sind seitdem das Bundesverfassungsgericht am 26. Februar 2014 die Drei-Prozent-Hürde für verfassungswidrig und nichtig erklärt hat. ${ }^{57}$ Auf Landes- und Regionalebene machte die Partei zuletzt aber eher durch Skandale von sich reden. In diesen Querelen schwingt nach wie vor eine ungeklärte Richtungsfrage mit, denn hinter der Anti-Euro-Position sammeln sich zunehmend auch rechtspopulistische Strömungen. Nicht auszuschließen ist, dass die AfD - ähnlich wie zuletzt die Piraten - an innerparteilichen Konflikten scheitert.

\section{Zusammenfassung und Ausblick}

Die Wahl 2013 stellt eine Zäsur dar, denn erstmals ist die FDP nicht im Deutschen Bundestag vertreten. Damit ist das seit der Vereinigung etablierte Fünf-Parteien-System erst einmal Vergangenheit. Die neue Vier-Parteien-Konstellation erscheint aber etwas ungleichgewichtig, denn die Union steht im 18. Bundestag drei Parteien gegenüber, die eher dem linken Lager zugerechnet werden. Ursache für diese Verschiebungen ist eine anhaltend hohe Volatilität mit zwei gegenläufigen Ausprägungen. Zum einen stieg die Bereitschaft des Elektorats, sich auch Parteien außerhalb des etablierten Fünf-Parteien-Spektrums zuzuwenden: Rund zehn Prozent der Wähler (die Stimmen für die FDP nicht eingerechnet) bevorzugten diesmal andere, kleinere Parteien, die Gefahr in Kauf nehmend, dass diese bei der Sitzverteilung am Ende keine Rolle spielen würden. Damit kontrastiert auffallend das Wiedererstarken der beiden großen Volksparteien, die erstmals seit langer Zeit gleichzeitig an Stimmen zulegten. Da der größte Teil dieser Zuwächse auf Wechselwählern aus demselben Lager beruht, führte dies - ob gewollt oder ungewollt - zu einer Stärkung des jeweiligen Zentrums. Die Bedeutung der Lager dürfte sich allerdings künftig deutlich abschwächen. Aufgrund ihrer konsequenten Modernisierungsstrategie hat Merkels CDU zentrale Konfliktpunkte mit den Grünen in der Energie- und Gesellschaftspolitik aus dem Weg geräumt. Auch wenn das Verhältnis zwischen Grünen und CSU auf Bundesebene ein potentieller Störfaktor bleibt, ergeben sich für beide Parteien daraus neue Koalitionsoptionen - die Zeiten schwarz-gelber oder rot-grüner Dominanz bei der Regierungsbildung auf Bundes- und Landesebene scheinen damit vorerst vorbei.

Offen bleibt, welche Konsequenzen das Übergewicht der Regierungsfraktionen im Bundestag für das Parteiensystem haben wird. Die letzte Große Koalition führte zumindest zu einem erheblichen Wählerabfluss, auch wenn das befürchtete Erstarken rechter oder populistischer Parteien ausblieb. Das muss nicht so bleiben, denn angesichts der bei der letzten Bundestagswahl gezeigten Offenheit vieler Wähler gegenüber neuen Parteien erscheinen Erfolge kleinerer Parteien nicht ausgeschlossen. Insbesondere die AfD könnte zu den Profi- 
teuren gehören, sollte es der Großen Koalition nicht überzeugend gelingen, die anstehenden Herausforderungen in Europa zu bewältigen. Ein Trend dürfte ungebrochen sein: die immer stärkere Personalisierung von Politik. Bereits in der vergangenen Wahlperiode waren nicht die im Koalitionsvertrag vereinbarten Maßnahmen prägend für die Regierungswahrnehmung; entscheidend war vielmehr die „Risikokompetenz, um auf politische Überraschungen angemessen zu reagieren" 58 - diese Kompetenz hat Merkel in der Bundespolitik in der Eurofrage und nach Fukushima ebenso bewiesen wie Seehofer in Bayern. Dieser hat seinen Rückzug für 2018 bereits angekündigt; ob Merkel die Union in eine vierte Wahlperiode führt, darf bezweifelt werden. „Natürliche“ Nachfolger bieten sich für beide derzeit nicht an. Vor diesem Hintergrund stellt sich die Ausgangsposition der SPD besser dar, denn Gabriels Position ist nach erfolgreichen Koalitionsverhandlungen und dem Mitgliedervotum stärker denn je; die jahrelang ungeklärte Führungsfrage scheint beigelegt.

Die letzte Wahlperiode war auch geprägt von der Forderung der Bürger nach mehr direkter Mitsprache, vor allem in Bereichen, die das unmittelbare Lebensumfeld betreffen (Stuttgart 21, Energienutzung beziehungsweise -versorgung). SPD und CSU wollten dem Rechnung tragen und eine entsprechende Willenserklärung im Koalitionsvertrag festschreiben. Die CDU hat dies mit dem Hinweis verhindert, es erscheine fraglich, ob die Bundesebene dafür der richtige Rahmen sei. Speziell bei der Lösung komplexer beziehungsweise globaler Probleme dürfte das Vertrauen der Bürger in die Instanzen der repräsentativen Demokratie tatsächlich relativ intakt sein. Mehr Erfolg versprechen die Ansätze für mehr Transparenz und Partizipation innerhalb der Parteien. Das Aufkommen der Piraten hat gezeigt, dass hier Defizite, aber auch Potenziale stecken, denn mit dem Versprechen von mehr Verfahrenstransparenz und Beteiligung haben sie viele politikferne jüngere Leute animiert, sich politisch zu betätigen. Und sie haben die etablierten Parteien aufgerüttelt, sich wieder mehr um Bürgernähe und um eine Aktivierung ihrer Mitglieder zu bemühen. Jüngste Beispiele - die Beteiligung der Mitglieder am grünen Wahlprogramm und das SPD-Mitgliedervotum - zeigten spektakuläre Erfolge. Das lässt hoffen, dass neben der Rekrutierung von politischem Spitzenpersonal auch das zuletzt eher vernachlässigte zweite Standbein von Parteien, die demokratische Willensbildung von unten nach oben, wieder gestärkt und damit auch die zunehmende Distanz zwischen Bürgern und Parteien abgebaut werden könnte.

58 Karl-Rudolf Korte / Niko Switek, a.a.O. (Fn. 17), S. 9. 